\title{
Social Capital and the Status Externality*
}

\author{
Jun-ichi Itaya ${ }^{\dagger} \quad$ Christopher Tsoukis ${ }^{\ddagger}$
}

April 22, 2020

\begin{abstract}
This paper investigates how the presence of social capital affects the externality arising from status-seeking preference as a parable for inefficient antagonistic behavior. It is assumed that the stock of social capital accumulates through the strategic interaction among rational, infinitely-lived, individuals of a finite number. Using a differential game, we show that there are two types of Markov perfect equilibrium strategies, of which one leads a society to zero social capital, while the other leads to the satiation level of social capital. When there is an unstable interior steady state, there is a threshold: with any initial stock of social capital above (below) that, society is able to build social capital (correspondingly, get stuck in a poverty trap of null social capital). In the latter case, the intervention of governments is called upon, because social welfare in the poverty trap is less than that in the social capital-rich society.
\end{abstract}

JEL classification: C72; D91; Z13

Keywords: social capital, status externality, poverty trap, Markov perfect equilibrium, differential game

$\pm$

${ }^{*}$ Earlier versions of the paper have been presented at the 19th International Conference of the Association for Public Economic Theory in Paris, July 2017 and the 73th Annual Congress of the International Institute of Public Finance in Tokyo, August 2017. We wish to thank Ronald Wendner and seminar participants for their useful comments. The first author gratefully acknowledges the financial support provided by the Grant-in-Aid for Scientific Research from the Japan Society for the Promotion of Science (16K03693).

${ }^{\dagger}$ Corresponding author. Graduate School of Economics and Business, Hokkaido University, Sapporo 0600809, Japan. Tel: +81-11-706-2858; Fax: +81-11-706-4947; E-mail: itaya@econ.hokudai.ac.jp

${ }^{\ddagger}$ Keele Business School, Keele University, Staffordshire, ST5 5AA, UK; Tel: +44 (0)1782 733101; E-mail: c.tsoukis@keele.ac.uk 


\section{Introduction}

Social capital is a concept with a long history in the social sciences, and the subject of a rapidly expanding literature in economics. In varying contexts, it has been shown to affect positively growth and development (Knack and Keefer, 1997; Knack and Zak, 1999; Grootaert and van Bastelaer, 2002; Putnam, Leonardi and Nanetti, 1993), institutional design and performance (Djankov, Glaeser, La Porta, Lopez-de-Silanes, and Shleifer, 2003) and financial development (Guiso, Sapienza and Zingales, 2004). Putnam (1993) found a correlation between civic engagement and governmental quality across Italian regions. LaPorta, Lopez-de-Silanes, Shleifer and Vishny (1999) found a cross-country correlation between trust and judicial efficiency (positive) and government corruption (negative). But, despite the lavish attention to the concept, a precise definition of it has remained elusive; this hampers both clear analytical development and empirical measurement. In the light of the above literature, questions such as: what are the main determinants of social capital accumulation?; when do societies build such capital and when do they get mired in social capital poverty traps?; does social capital enhance societal welfare?; when is active public policy necessary in order to encourage such accumulation?; are now recognized as key for growth and development. Our contribution is a formal analysis of these questions based on a clear definition of social capital.

Following landmark contributions in sociology(Coleman, 1990; Putnam, 1993, 2000; Fukuyama, 1997), another strand of literature places shared norms, beliefs or trust and social or governance structures that facilitate economic (and wider) activity and promote cooperation and efficiency, at the heart of social capital; Putnam, Leonardi, and Nanetti (1993), Bowles and Gintis (2002) and Routledge and von Amsberg (2002) are in this category. However, defining social capital by its supposed effect (cooperation) seems like putting the cart in front of the horses; a clear definition should go first, then the effects investigated. Additionally, in some of these analyses, social capital is a by-product of exchange, social interaction or other decision such as location; there is no associated investment decision. ${ }^{1}$

In an important early contribution, Glaeser, Laibson and Sacerdote (2002) model the

${ }^{1}$ Useful surveys on social capital include Durlauf and Fafchamps (2005) and Guiso, Sapienza and Zingales (2012). 
investment-into-social capital decision based on individual optimization. However, they define social capital as 'social characteristics that yield a market as well as non-market yield' (Glaeser et al., 2002, p. F439); as they themselves acknowledge however, such social skills (to use the everyday term) include those of '. . . the stereotypical used car salesman who has lots of individual social capital (i.e., he's good at selling lemons to naïve consumers), but who generates little net social capital because of his negative social capital externalities'. Thus, this type of skill should be more appropriately considered as a socially-related, private skill or form of human capital; social capital properly defined should be closer to what Glaeser et al. (2002) call aggregate social capital, which nets out all the externalities from individual capital; and which they treat as exogenous.

Other analyses (Guiso, Sapienza and Zingales, 2012; Durlauf and Fafchamps, 2005) emphasize the potential ability of social capital to alleviate negative externalities such as the free rider problem. While these contributions are in the right direction, the beneficial effects, often beliefs or values, arise within groups, social networks and associations; this appears restrictive in that beneficial collective action, even informal and unintended, may happen in the wider community. Additionally, there may be an element of circularity in such definitions, as 'networks' are often defined by shared norms.

This is not to deny that each of these analyses makes an important contribution; but a clear, synthetic definition is missing. Building on these, to alleviate ambiguities, we propose the following definition:

\section{Definition:}

'Social capital is the set of all community-related formal and informal engagements, beliefs, values, norms and practices that:

(a) help society achieve Pareto-superior outcomes, and

(b) require resources (time and/or effort) to build.'

We argue that this definition, alone, satisfies Solow's (2000) four criteria of a definition of capital: conceptual clarity, conscious investment, opportunity cost, depreciation. It is also quite general. We show that such a concept yields novel insights, thus satisfying the additional criterion of usefulness.

Manifestations of social capital may include contributions to charity, ethical behavior and norms such as politeness, mutual help and respect of the law, environmentally friendly norms 
and practices, quality of governance and institutions (Tabellini, 2008), etc. In all cases, there is 'something' in the background that motivates these types of behavior; to be built, this intangible form of capital requires investment such as formal and informal education and/or personal involvement and time. The beneficial effects arise from the alleviation of inefficiencies related to status-seeking, conspicuous, corrupt or aggressive behavior, other rat races, free-riding, etc. ${ }^{2}$

Other formal analyses of social capital include Chou (2006) in which social capital facilitates standard human capital formation in economic growth models. Such an economy has two possible steady states: of which, one is a stable social capital-poor equilibrium (a 'social capital poverty trap'), while the other is an unstable social capital-rich equilibrium. However, the emergence of a poverty trap stems from a non-concave reduced-form function of social capital which implicitly embodies the social capital spillover. We eschew this assumption. Antoci et al. (2007) study the co-evolution of social participation and social capital accumulation using an evolutionary game model ignoring accumulation of physical and human capital. They also find two dynamic attractors of the replicator dynamics in terms of the population of participants into social activities, one of which may be thought of as a social poverty trap because it entails a low steady state level of social capital. However, they have assumed a continuum of identical and bounded rational individuals, and a binary choice of time allocation which we will assume away. Closer to the spirit of our analysis is the paper by Growiec and Growiec (2014); they employ a representative agent framework in which multiple equilibria emerge as a result of complementarity between social capital and trust. Trust is also the theme in the overlapping generations framework of Varvarigos and Xin (2015), which analyses the joint dynamics of trust and the process of physical capital accumulation. Multiple and path-dependent steady-state equilibria arise here, too. Room for improvement over these interesting analyses is provided by their focus on trust; as mentioned, our definition of social capital is more general.

\footnotetext{
${ }^{2}$ In some analyses, social capital may confer utility directly, e.g., in Helliwell, Aknin, Shiplett, Huang and Wang (2017), due to a lower crime rate or greater social ties and similar effects. These effects are akin to the 'warm glow' type of benefit from giving (Andreoni, 1990) except that they stem from the level of social capital rather than the individual contributions to it. In our analysis, we ignore the private benefits from individual investment in social capital as we want to focus on the deeper issue of how the stock of social capital mediates social interactions with others.
} 
We advance the existing literature in several ways. Our major contribution is a novel formal framework based on social capital as a community-related state variable in the form of a public good, rather than a macroeconomic aggregative variable. It is gradually formed through costly individual contributions and provides benefits in a way that alleviates the social inefficiencies associated with negative externalities in a community consisting of a $f$ nite number of individuals. The negative externalities arise from status-seeking competition among individuals endowed with status-seeking preferences, which lead every individual to welfare-decreasing overconsumption. However, as we explain below, such externalities are used only as an example of a wider class of 'un-sociable' (inefficient) behavior. In addition to the negative externalities arising from status-seeking competition, we also capture the dynamic strategic considerations regarding social capital accumulation among a finite number of forward-looking and fully-rational individuals using a differential game. This treatment of social capital also highlights not only the tendency of free-riding of individuals to avoid own costly investments but also stock externalities, whose benefits spill over intertemporally and intratemporally across many individuals and work by discouraging wasteful or excessive consumption; that is, in our setting, a finite number of individuals explicitly recognize how these externalities affect their own as well as others' behavior, unlike most of the above-mentioned contributions which are based on the representative agent setting in conjunction with a continuum of agents. This modeling strategy, therefore, generalizes the previous single- or representative agent optimizing models based on a continuum of individuals. In order to focus on the formation of social capital without unnecessary complications, the model involves simple microfoundations, based on a finite number of infinitely-lived, forward-looking and rational individuals with flexible labor who are able to explicitly recognize their interaction with one another; it is static except for the evolution of social capital which induces dynamics, thus ignoring the formation of physical and human capitals for tractability. Investment in social capital relies on individual contributions of time; this time is costly in an opportunity-cost sense and, implicitly, enhances social activities such as formal and informal education. Social capital is also subject to gradual depreciation (implicitly, this may arise from the residential mobility of individuals, as e.g., in Glaeser, et al., 2002; Antoci et al., 2007). In such a simple dynamic game setting, we find the solution to the formation of social capital as a Markov perfect equilibrium which entails several desirable properties 
such as subgame perfectness and no commitment by allowing players to immediately react to changes in the current state of the system (i.e., the current stock level of social capital).

As already mentioned above, to capture inefficiencies as well as to highlight the beneficial aspects of social capital, the utility function exhibits a status motive (alternatively known as 'keeping up with the Joneses') that induces zero-sum behavior (see, e.g., among many others: Alonso-Carrera, Caballé and Raurich, 2004; Alvarez-Cuadrado, Monteiro, and Turnovsky, 2004; Futagami and Shibata,1998; Tsoukis, 2007; Tournemaine and Tsoukis, 2013). But the emphasis on status is made only for analytical convenience; very similar behavior and effects includes corruption and aggression, ostentatious behavior, rat races, as well as free-riding. Our framework is perfectly compatible with this wider interpretation, in which social capital restrains individuals from any unsociable (i.e., inefficient) behavior.

We derive several results related to equilibrium labor supply, social capital and welfare in prisoner's dilemma-type of situations with repeated interaction. In this regard, we reach an optimistic conclusion in that, under plausible conditions, the accumulation of social capital is likely to autonomously and spontaneously take place thus leading to a Pareto-improving allocation. In this case, there exist (i.e., a continuum of) MPE strategies which monotonically lead the society to its satiation level. However, either when the disutility of labor supply is weaker, or when the number of a community is large, or when the wage rate is lower, overconsumption tends to prevail so that an interior steady state is present but dynamically unstable, while at the same time the two but non-interior long run equilibria are also present as an absorbing set. That is, the society converges to either a long-run equilibrium characterized by zero social capital or that characterized by the satiated level of social capital. In particular, if the initial level of social capital is low, overconsumption is more likely to occur thereby leading to zero social capital; consequently, a social poverty trap emerges as well. In this pessimistic case, a situation akin to a prisoners' dilemma arises because the negative externality arising from status-seeking competition overweighs the positive externality arising from social capital accumulation. As a result, the society fails to accumulate social capital, and ends up in a situation of a 'social poverty trap' in which there is no social capital stock and the economy is unable to escape from it. Due to the inverse relationship between social capital and private consumption we find, moreover, the welfare of individuals at the social poverty trap is less than that at the satiated level of social capital. In addition, there can 
exist multiple or a continuum of Markov perfect Nash equilibrium strategies (MPE) which follow different paths towards the long run equilibria stated above, so that the initial choices of consumption levels also play a critical role in determining long run outcomes. The multiplicity of MPE strategies create the possibility of inefficient coordination failure. Since the social poverty trap with overconsumption is Pareto-dominated by the long run equilibrium with a satiation level of social capital, all individuals are better off coordinating on the initial choice of consumption at the outset of the game. The policy implication of this study is that societies can benefit from coordinating on the equilibrium path starting from lower initial consumption towards the long run equilibrium with a satiation level of social capital stock; this can be achieved either by aligning expectations of private agents through communication, campaign, public announcement, education and so on, or by some type of government intervention such as consumption taxation.

The organization of the paper is as follows. The next section describes the basic model, and then characterizes the dynamics of the model. Section 3 makes a welfare comparison between the command economy and the decentralized economy. Section 4 concludes the paper with a discussion and suggestions for future research. Additional mathematical proofs will be given in Appendices A, B, and C.

\section{The Model}

There are $n(\geq 2)$ individuals. The instantaneous utility of individual $i$ depends positively on individual consumption, $c_{i}$, and social status $c_{i} / \bar{c}$, where $\bar{c}=\left(\sum_{j=1}^{n} c_{j}\right) / n$ represents average consumption. The variable $S$ represents social capital; as mentioned, it is a stock variable that accumulates over time through each individual's contribution through purposeful social activity such as charity, volunteer work, civic engagement, mutual help, or simple acts of goodwill and courtesy. Our definition is quite general and encompasses the various formulations proposed in the literature; in particular, this activity and contribution can be done formally or informally, through membership of related associations, groups and networks or individually. The objective function of individual $i$ is a discounted-sum of utilities over an infinite-time horizon:

$$
u_{i}=\int_{0}^{\infty}\left[\log c_{i}-\beta l_{i}+\left(1-\theta_{i}(S)\right) \log \left(\frac{c_{i}}{\bar{c}}\right)\right] e^{-\rho t} d t, \quad \rho>0, \quad i=1,2, \ldots, n
$$


where the function $\log \left(c_{i} / \bar{c}\right)$ captures the status externality, and $\beta$ represents the weight on the disutility of labor. The third term in (1) captures the fact that the utility of each individual increases in their consumption relative to average; an effect commonly referred to as status-seeking (or 'keeping up with the Joneses' or 'rat race'). ${ }^{3}$ In addition, the variable $\theta_{i}(S)$ represents the perception of social capital by individual $i: \theta_{i}(S): R_{+} \mapsto[0,1]$; furthermore, $\theta_{i}(S)$ is an increasing function of $S$. The variable $\theta_{i}($.$) determines the extent to which the$ status externality is internalized due to the spirit of social capital, and consequently, the weight put on the status-seeking motive, $1-\theta_{i}(S)$, is a decreasing function of $S$. This formulation means that the higher is the social capital stock $S$, the lower is the individual's incentive to "out-do others" in terms of relative consumption; by the same token, if social capital is accumulated through time, the incentive to 'out-do others' is weakened. To get a closed-form solution, we further impose a special form on the function $\theta(S)$ such as

$$
\theta(S)= \begin{cases}\theta S & \text { if } S<\bar{S} \\ 1 & \text { if } \bar{S} \leq S\end{cases}
$$

where the parameter $\theta$ is assumed to be the same to all individuals. We call $\bar{S}(=1 / \theta)$ 'the satiation level of the social capital stock' perceived by every individual.

Individuals decide at each point in time how much they engage in social activity as well as how much to consume. Their choices of consumption are constrained by income, which is earned from working at a constant wage rate $w$. Accordingly, individual $i$ is subject to the following flow budget constraint at each point in time:

$$
c_{i}=w l_{i}
$$

For notational simplicity we set the wage rate equal to 1 in what follows. In addition, individual $i$ is also subject to the time constraint:

$$
1 \geq l_{i}+a_{i}
$$

\footnotetext{
${ }^{3}$ Although it appears that larger $S$ reduces the utility in (1), this is not the case. Since the presence of status-seeking preferences usually leads individuals to overconsumption which ends up deteriorating their well-being, the tendency of overconsumption compared to the Pareto-efficient level is gradually weakened as a result of the accumulation of social capital, thereby generating positive externalities on individual utility.
} 
where $a_{i}$ is the time allocated to social activities, which contributes to accumulating the stock of social capital over time, $l_{i}$ is the amount of labor supply and the initial time endowment is normalized to be 1 . Combining both constraints yields

$$
c_{i}=1-a_{i}
$$

The aggregate stock of social capital will continuously change over time according to

$$
\dot{S}=S\left(\sum_{i=1}^{n} a_{i}\right)-\delta S
$$

where $\delta$ is a constant depreciation rate of social capital $(0<\delta<1)$. The input to social capital depends on the product of $S$, a manifestation of the spillover effects of the existing social capital stock on itself, times the total amount of individual contributions to it.

We consider an $n$-player differential game in which every individual maximizes the intertemporal utility $u_{i}$ in (1) by selecting the time paths of $c_{i}$ and $a_{i}$ subject to (3), (4), and the given initial level of social capital, $S_{0}(\geq 0)$, taking into account the others' strategies $a_{j}(j=1,2, \ldots, n: j \neq i)$. The game is symmetric and stationary as all individuals' instantaneous utility functions and feasible sets, and their influence on the state's evolution (4) are identical, and not dependent explicitly on time.

\subsection{Solution Concept}

We solve the above-mentioned differential game using the notion of a stationary Markov perfect equilibrium (MPE), which is appealing because, in ruling out all direct strategic interactions, it allows us to use optimal control tools. We first rewrite the differential game as the one in which at each moment in time, $t$, each individual selects a single control $c_{i}(t)$ rather than the two control variables $c_{i}(t)$ and $l_{i}(t)$, which can be done by using (3). Moreover, following Dockner et al. (2000) and Rowat (2006) and taking into the fact that the maximization of individual $i$ 's payoff (1) depends on the own $c_{i} \equiv \phi^{i}$, the others' strategies $\left(\phi^{1}, \phi^{2}, ., \phi^{i-1}, \phi^{i+1} . ., \phi^{n}\right)$ and the initial condition $S_{0}$, the game $\Gamma\left(S_{0}, 0\right)$ can be formulated 
as an optimal control problem:

$$
\begin{aligned}
V_{i}\left(S_{0}\right) & \equiv \max _{\left\{c_{i}(t)\right\}_{0}^{\infty}} \int_{0}^{\infty}\left[\log c_{i}(t)-\beta c_{i}(t)+(1-\theta S(t)) \log \left(\frac{c_{i}(t)}{\bar{c}}\right)\right] e^{-\rho t} d t,(5) \\
\text { subject to } \dot{S}(t) & =S(t)\left[1-c_{i}(t)+\Sigma_{j \neq i}\left(1-\phi^{j}(S(t))\right]-\delta S, S(0)=S_{0} \in[0,1 / \theta],\right. \\
c_{i}(t) & \in[0,1], \text { and } \bar{c}=\frac{c_{i}(t)+\Sigma_{j \neq i} \phi^{j}(S(t))}{n},
\end{aligned}
$$

where the reason why the others' choices of consumption, $\phi^{j}(S(t))$, are functions of the current stock level of aggregate social capital, $S(t)$, alone will be stated below. The variable $t$ is omitted except when it is strictly necessary.

To state the concept of MPE in a mathematically rigorous way, we reproduce a series of definitions given by Dockner et al. (2000) with notational modifications:

Definition 1 (Dockner et al., 2000, Definition 3.1). A control path $c_{i}:[0, \infty) \mapsto \mathbb{R}_{+}$is feasible for the game $\Gamma\left(S_{0}, 0\right)$ if the initial value problem defined by (5) has a unique, absolutely continuous solution $S($.$) such that the constraints S(t) \in[0,1 / \theta]$ and $c_{i}(t) \in[0,1]$ hold for all $t$ and the integral in (1) is well defined.

As the game $\Gamma\left(S_{0}, 0\right)$ is stationary in the present setting, we can focus on equilibria supported by stationary strategies. Hence, stationary Markov strategies are functions only of the current state. Then we can define:

Definition 2 The $n$-tuple of functions $\left(\phi^{1}, \phi^{2}, \ldots, \phi^{n}\right)$ is a stationary Markov Nash equilibrium if for each $i \in\{1,2, . ., n\}$ an optimal control path $c_{i}(t)$ of the problem $\Gamma\left(S_{0}, 0\right)$ exists and is given by the stationary Markov strategy $c_{i}(t)=\phi^{i}(S(t))$.

We use the further strengthening of Markov Nash equilibrium, that is, subgame perfectness, to characterize an equilibrium path:

Definition 3 (Dockner et al., 2000, Definition 4.4). The $n$-tuple of functions $\left(\phi^{1}, \phi^{2}, \ldots, \phi^{n}\right)$ is a Markov Nash equilibrium of the game $\Gamma\left(S_{0}, t\right)$. The Markov Nash equilibrium is Markov perfect equilibrium (MPE) if for each $(S, t) \in \mathbb{R}_{+} \times[0, \infty)$, the subgame $\Gamma(S, t)$ admits a Markov Nash equilibrium $\left(\psi^{1}, \psi^{2}, \ldots, \psi^{n}\right)$ such that $\psi^{i}(S, s)=\phi^{i}(S, s)$ for all $i \in\{1,2, \ldots, n\}$ and all $(S, s) \in \mathbb{R}_{+} \times[t, \infty)$. 
Since the subgame $\Gamma(S, t)$ is stationary, $\Gamma(S, 0)=\Gamma(S, t)$ and thus all stationary Markov Nash equilibria are MPE; consequently, $\psi^{i}(S, s)=\phi^{i}(S, s)$ in Definition 3 is replaced by $\psi^{i}(S(t))=\phi^{i}(S(t))$ for all $i \in\{1,2, \ldots, n\}$ and all $t \in[0, \infty)$ in the present setting. We also make the following assumption to guarantee the differentiability of the value function $V_{i}(S)$ :

Assumption 1: The value function of individual $i, V_{i}(S)$ is Lipschitz continuous.

\subsection{Candidate Markov Perfect Equilibrium Strategies}

After substitution of $l_{i}=c_{i}$ in (1), the value function (5) satisfies the Hamilton-JacobiBellman (HJB) equation such that

$\rho V_{i}(S)=\max _{\left\{c_{i}\right\}}\left\{\log c_{i}-\beta c_{i}+(1-\theta S) \log \left(\frac{c_{i}}{C(S) / n}\right)+V_{i}^{\prime}(S)\left[S \sum_{j=1, j \neq i}^{n}\left(1-c_{i}-\phi^{j}(S)\right)-\delta S\right]\right\}$

for all $t \geq 0$, where $C(S) \equiv c_{i}+\sum_{j=1, j \neq i}^{n} \phi^{j}(S)$. In solving the maximization problem defined in the right-hand-side of (6) (which amounts to finding Markov Nash equilibrium strategies) at each moment in time, the $i$-th individual takes the other players' optimal strategies, $\left\{\phi^{j}(S)\right\}_{j \neq i}(j=1,2, . ., n)$, as given. The first-order condition for $i$ 's optimal choice of consumption is given by

$$
\frac{1}{c_{i}}-\beta+(1-\theta S) \frac{1}{c_{i}} \frac{\sum_{j=1, j \neq i}^{n} \phi^{j}(S)}{C(S)}-V_{i}^{\prime}(S) S\left\{\begin{array}{l}
=0 \Longrightarrow c_{i} \in(0,1] \\
>0 \Longrightarrow c_{i}=1
\end{array}\right.
$$

According to (7), each individual, when choosing $c_{i}$, trades off the marginal utility gains from an increase in current consumption (i.e., $1 / c_{i}$ ) and the associated status-seeking consumption (which is captured by the term $\Sigma_{j=1, j \neq i}^{n} \phi^{j}(S) / C$ ) against the sum of the utility loss from increased labor supply (i.e., $\beta$ ) and the marginal loss in the discounted value of the future stream of intertemporal externalities arising from a decrease in the social capital stock (which is captured by the term $V_{i}^{\prime}(S) S$ ). If the marginal utility from an increase in current consumption (i.e., the first three components in (7)) is larger than the payoff loss implied by the decrease in $a_{i}$ (i.e., $V_{i}^{\prime}(S) S$ in (7)) for all levels of $c_{i} \in(0,1]$, then $i$ will rationally devote all resources to current consumption. However, $i$ never chooses $c_{i}=0$ due to the property of logarithmic utility functions. Moreover, a close inspection of (7) also reveals the following features; the higher the disutility of labor (i.e., higher $\beta$ ), the lower the labor supply and 
thus the lower the instantaneous consumption due to the budget constraint (3); on the other hand, instantaneous consumption is stimulated by status-seeking competition. Finally, the accumulation of social capital generates positive externalities as it deters overconsumption by discouraging the incentive to 'out-do others' through lowering the weight $(1-\theta S)$.

The envelope condition is given by

$$
\begin{gathered}
\rho V_{i}^{\prime}(S)=-\theta \log \left(\frac{c_{i}}{C(S) / n}\right)+(1-\theta S) \frac{\left(-\Sigma_{j=1, j \neq i}^{n} \phi^{j^{\prime}}(S)\right)}{C(S)}+ \\
V_{i}^{\prime \prime}(S)\left[S \Sigma_{j=1, j \neq i}^{n}\left(1-c_{i}-\phi^{j}(S)\right)-\delta S\right]+ \\
V_{i}^{\prime}(S)\left[\Sigma_{j=1, j \neq i}^{n}\left(1-c_{i}-c_{j}(S)\right)+S \Sigma_{j=1, j \neq i}^{n}\left(-\phi^{j \prime}(S)\right)-\delta\right] .
\end{gathered}
$$

Since the present game is a symmetric one, a natural focus is placed on symmetric equilibria. This focus allows us not only to drop the subscripts $i, j$ in the subsequent discussion, but also to make use of the facts that $\log (c /(C(S) / n))=\log 1=0$; consequently, the first order condition (7) can be reduced to

$$
\frac{1}{c}+(1-\theta S) \frac{n-1}{c n}=\beta+V^{\prime}(S) S .
$$

which gives the symmetric Markov Nash equilibrium (i.e., Markov perfect equilibrium) strategies $c=c_{i}=\phi^{j}(S), j=1,2, . ., n$ and $j \neq i$. Substituting (9) into the right-hand-side of the symmetric form of (8), together with $c=c_{i}=\phi^{j}(S), j=1,2, . ., n$ and $j \neq i$, results in

$$
\begin{aligned}
\rho V^{\prime}(S)= & {\left[\frac{1}{c(S)}-\beta\right] c^{\prime}(S)+V^{\prime \prime}(S)[S n(1-c(S))-\delta S] } \\
& +V^{\prime}(S)\left[n(1-c(S))-S n c^{\prime}(S)-\delta\right] .
\end{aligned}
$$

After some tedious manipulations, we can rewrite (10) as follows (see Appendix A):

$$
\begin{gathered}
c^{\prime}(S)= \\
-\frac{\rho\left[1-\beta c(S)+(1-\theta S) \frac{n-1}{n}\right] S^{-1}+\theta \frac{n-1}{n}[n(1-c(S))-\delta]}{\frac{1}{c(S)}[(n-c(S))-\delta]\left[1-\beta c(S)+(1-\theta S) \frac{n-1}{n}\right]+(1-\theta S) \frac{n-1}{n}+\beta[n(1-c(S))-\delta]} .
\end{gathered}
$$




$$
\frac{d c}{d S}=\frac{1}{[\theta(n-1) S+\beta \rho]^{2}}\left(\frac{1}{n} \theta \rho(1-n)(2 n-1+\beta(-n+\delta+\rho))\right)<0 \text { if } \beta<\frac{2 n-1}{n-\delta-\rho}
$$

\subsection{Dynamics and Steady State}

We will draw the representatives of Markov strategies in a control and state space in order to characterize qualitative solutions to the nonlinear ordinary differential equation (11). The solutions satisfying (11) are only candidates and, in some cases, many of them do not qualify for the globally defined MPE strategies studied by Rowat (2007). In order to qualify for the globally defined MPE satisfying the properties stated later, those solutions must pass further tests. To stress the candidate status, we use a notation, $\phi(S)$, instead of $c(S)$. Let us first denote by $C_{2}$ the locus where $\phi^{\prime}(S)$ goes to plus/minus infinity, and by $C_{3}$ the locus where $\phi^{\prime}(S)$ equals zero in the $(S, c)$ space:

$$
\begin{aligned}
& C_{1}:=\{(S, c): \dot{S}=\operatorname{Sn}(1-\phi(S))-\delta S=0\} \\
& C_{2}:=\left\{(S, c): \phi^{\prime}(S) \rightarrow \pm \infty\right\} \\
& C_{3}:=\left\{(S, c): \phi^{\prime}(S)=0\right\}
\end{aligned}
$$

First, we can obtain the steady state line $C_{1}$ by setting $\dot{S}=0$ in the symmetric form of (4); that is,

$$
c=1-\frac{\delta}{n},
$$

which implies that the steady-state line $C_{1}$, denoted by $\dot{S}=0$ in Fig. 1, is a horizontal line at $c=1-(\delta / n)$ in the $(S, c)$ space. The arrows illustrated in Fig. 1 indicate that for initial values of $\phi(S)$ above the steady state locus, $c=1-(\delta / n)$, the social capital stock is declining in time, while for those of $\phi(S)$ below the steady state locus, the social capital stock is increasing in time. It is also important to note that there is an upper bound on $S$, as illustrated by the dotted vertical straight line at $1 / \theta$ in Fig. 1 (which is called 'the satiation locus'). Beyond this upper bound, there is no incentive for any individual to accumulate more social capital, because further social capital accumulation reduces utility (i.e., if $S>1 / \theta$, $1-\theta(S)<0$ in $(1))$. 


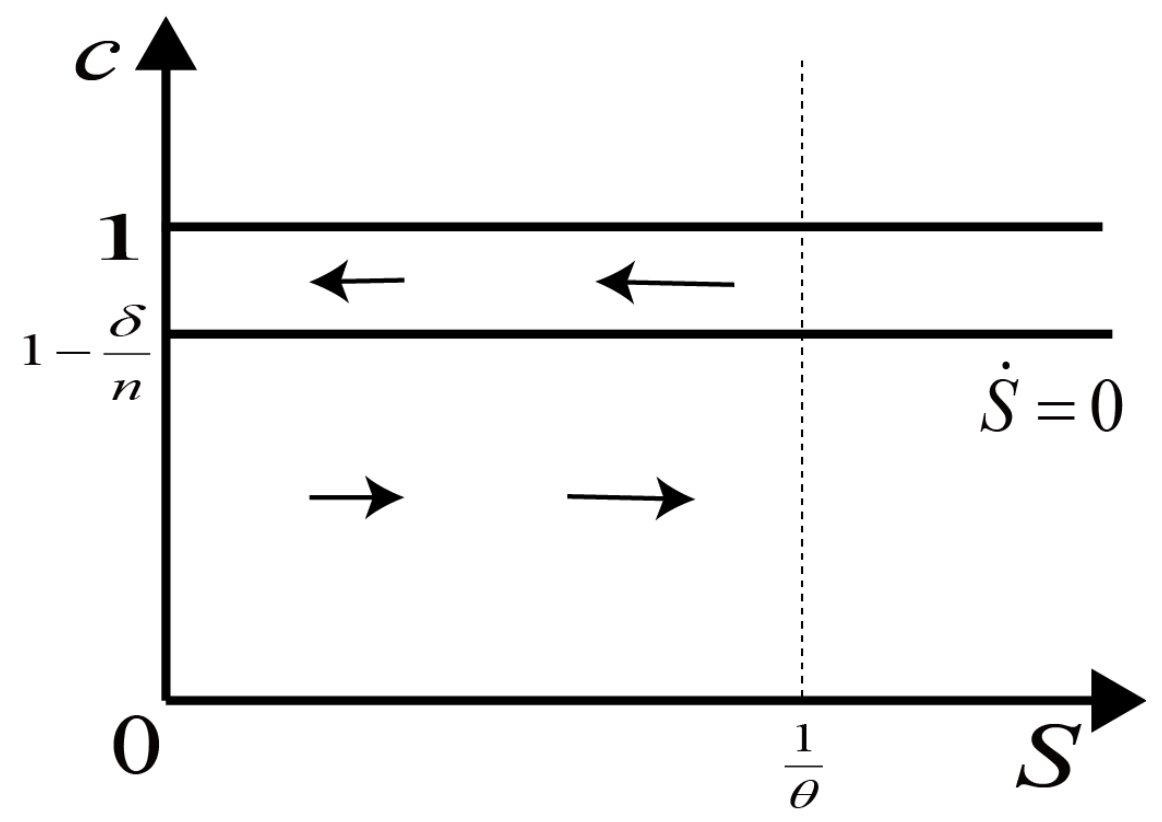

Figure 1: Steady state locus

Next, by setting the denominator in (11) equal to zero, we can obtain the locus of the curve $C_{2}$ :

$$
\frac{1}{c}[(n-c)-\delta]\left[1-\beta c+(1-\theta S) \frac{n-1}{n}\right]+(1-\theta S) \frac{n-1}{n}+\beta[n(1-c)-\delta]=0,
$$

which we call 'the non-invertibility locus' following Rowat (2007). After multiplying $c$ on both sides of (14) and some manipulation, we can rewrite (14) as follows:

$$
\beta c^{2}(1-n)-c+(n-\delta)\left[1+(1-\theta S) \frac{n-1}{n}\right]=0, \text { with } c \neq 0,
$$

whose solution for $c$ is given by: ${ }^{4}$

$$
c=\frac{\sqrt{1+4 \beta(n-1)(n-\delta)\left[1+(1-\theta S) \frac{n-1}{n}\right]}-1}{2 \beta(n-1)},
$$

\footnotetext{
${ }^{4}$ Since another root of the quadratic equation (15) is negative, it is irrelevant because the consumption
} level $c$ must be nonnegative. 
which entails that

$$
\begin{aligned}
\lim _{S \longrightarrow 0} c & =\frac{\sqrt{1+4 \beta(n-1)(n-\delta) \frac{2 n-1}{n}}-1}{2 \beta(n-1)} \gtreqless 1, \text { and } \\
0 & <\lim _{S \longrightarrow 1 / \theta} c=\frac{\sqrt{1+4 \beta(n-1)(n-\delta)}-1}{2 \beta(n-1)}<1 .
\end{aligned}
$$

An important feature to be noted is that if the solution curve of $\phi(S)$ crosses the curve $C_{2}$, it becomes discontinuous at that intersection point; consequently, it is ruled out as a solution (see the next subsection for further details).

Further inspection of (16) reveals not only that the curve $C_{2}$ may cross the vertical axis (hence, $S=0$ ) above or below the budget constraint $c=1$ according to

$$
\frac{\sqrt{1+4 \beta(n-1)(n-\delta) \frac{2 n-1}{n}}-1}{2 \beta(n-1)}-1 \gtreqless 0 \text { if and only if } \beta \lesseqgtr \frac{(n-\delta)(2 n-1)-n}{n(n-1)},
$$

but also that the curve $C_{2}$ may cross the vertical axis (hence, $S=0$ ) above or below the steady state line $1-(\delta / n)$ according to

$$
\frac{\sqrt{1+4 \beta(n-1)(n-\delta) \frac{2 n-1}{n}}-1}{2 \beta(n-1)}-\left(1-\frac{\delta}{n}\right) \gtreqless 0 \text { if and only if } \beta \lesseqgtr \frac{2 n}{n-\delta} .
$$

Lastly, the curve $C_{2}$ may cross the satiation locus $\bar{S}=1 / \theta$ above or below the steady state line $1-(\delta / n)$ according to

$$
\frac{\sqrt{1+4 \beta(n-1)(n-\delta)}-1}{2 \beta(n-1)}-\left(1-\frac{\delta}{n}\right) \gtreqless 0 \text { if and only if } \beta \lesseqgtr \frac{n}{n-\delta} .
$$

Taking into account of (17), (18), (19), and (20) in conjunction with the following facts:

$$
\begin{aligned}
\frac{(n-\delta)(2 n-1)-n}{n(n-1)}-\frac{n}{n-\delta} & =\frac{n^{3}-4 n^{2} \delta-n^{2}+2 n \delta^{2}+3 n \delta-\delta^{2}}{n(n-1)(n-\delta)} \gtreqless 0, \\
\frac{(n-\delta)(2 n-1)-n}{n(n-1)}-\frac{2 n}{n-\delta} & =\frac{-\delta\left(\delta-3 n+4 n^{2}-2 n \delta\right)}{n(n-1)(n-\delta)}<0, \text { and } \\
\frac{2 n-1}{n}-\frac{n}{n-\delta} & =\frac{n^{2}-(2 \delta+1) n+\delta}{(n-\delta) n} \gtreqless 0,
\end{aligned}
$$

we can draw several geometric representations for the curve $C_{2}$ in Figs. $2-9$, corresponding to various parameter values of $n, \beta$ and $\delta$. 
On the other hand, the curve $C_{3}$ is obtained by setting the numerator in (11) equal to zero. Solving for $c$ gives the following locus:

$$
c=\frac{(n-\delta-\rho) \theta \frac{n-1}{n} S+\rho \frac{2 n-1}{n}}{\rho \beta+\theta(n-1) S},
$$

with

$$
\lim _{S \rightarrow 0} c=\frac{2 n-1}{\beta n} \text { and } \lim _{S \rightarrow \frac{1}{\theta}} c=\frac{(n-\delta) \frac{n-1}{n}+\rho}{\rho \beta+(n-1)} .
$$

Taken together, it turns out that the curve $C_{3}$ is an orthogonal hyperbolic curve in the $(S, c)$ space which intersects the vertical axis at point $(0,(2 n-1) / \beta n)$, as illustrated in Figs. $2-9$. This intercept of the curve $C_{3}$ may lie above or below the intercept of the budget constraint $c=1$ on the vertical axis (i.e., $S=0$ ) according to

$$
\frac{2 n-1}{\beta n}-1=\frac{(2-\beta) n-1}{\beta n} \gtreqless 0 \text { if and only if } \beta \lesseqgtr \frac{2 n-1}{n} \text {, }
$$

while the curve $C_{3}$ may cross the vertical axis (hence, $S=0$ ) above or below the steady state line $1-(\delta / n)$ according to

$$
\frac{2 n-1}{\beta n}-\left(1-\frac{\delta}{n}\right)=\frac{2 n-n \beta+\beta \delta-1}{\beta n} \gtreqless 0 \text { if and only if } \beta \lesseqgtr \frac{2 n-1}{n-\delta} .
$$

Lastly, the curve $C_{3}$ may cross the satiation locus $1 / \theta$ above or below the steady state line $1-(\delta / n)$ according to

$$
\frac{(n-\delta) \frac{n-1}{n}+\rho}{\rho \beta+(n-1)}-\left(1-\frac{\delta}{n}\right)=\rho \frac{-(n-\delta) \beta+n}{[\rho \beta+(n-1)] n} \gtreqless 0 \text { if and only if } \beta \lesseqgtr \frac{n}{n-\delta} .
$$

Collecting the arguments discussed so far, we draw several geometric representations for the curves $C_{2}$ and $C_{3}$, depending on the combinations of various parameter values of $\beta, n$ and $\delta$. Four remarks are in order. First, based on the information regarding the signs of (11) which are determined according to the respective regions divided by the curves $C_{2}$ and $C_{3}$, together with the signs indicated by Fig.1, we can construct several phase diagrams indicating how the variables $c$ and $S$ evolve, as illustrated in Figs. $2-9 .{ }^{5}$ Second, it is important to note that not all strategies satisfying (11), which are drawn in these figures, are globally defined

\footnotetext{
${ }^{5}$ To draw the curves $C_{2}$ and $C_{3}$, we first check whether the intercept of curve $C_{2}$ on the vertical axis may
} 
MPE strategies. In the next subsection, we will provide further conditions to pin down the globally defined MPE strategies characterizing optimal consumption paths. ${ }^{6}$ Third, there is a singular point $(0,(2 n-1) / \beta n)$ on the vertical axis at which both the numerator and denominator in (11) are equal to zero at the same time. As a result, there are a continuum of strategies $\phi_{1}$ which either emit from this singular point or converge to it, as seen in Figs. $2-9$. Fourth, note also that as $S$ approaches zero, following the strategy $\phi_{3}$, the associated level of consumption tends to infinity due to the feature of (11) and thus it will inevitably hit the budget constraint $c=1$.

be greater or less than that of curve $C_{3}$. To do this, we take the difference between their intercepts to yield

$$
\frac{\sqrt{1+4 \beta(n-1)(n-\delta) \frac{2 n-1}{n}}-1}{2 \beta(n-1)}-\frac{2 n-1}{\beta n}=\frac{\sqrt{1+4 \beta(n-1)(n-\delta) \frac{2 n-1}{n}}-1-2(2 n-1)\left(1-\frac{1}{n}\right)}{2 \beta(n-1)}
$$

whose numerator can be manipulated by squaring both sides of the above expression to yield:

$$
1+4 \beta(n-1)(n-\delta) \frac{2 n-1}{n}-\left[1+2(2 n-1)\left(1-\frac{1}{n}\right)\right]^{2} \gtreqless 0 \text { if and only if } \beta \gtreqless \frac{2(n-1)+(1 / n)}{n-\delta} .
$$

The above result indicates that as long as $\beta>[2(n-1)+(1 / n)] /(n-\delta)$, the intercept of curve $C_{2}$ on the vertical axis $S=0$ is located above that of the curve $C_{3}$. On the other hand, we can also check whether the intercept of the curve $C_{2}$ with the satiation locus $1 / \theta$ may be greater or less than that of curve $C_{3}$ according to

$$
\frac{\sqrt{1+4 \beta(n-1)(n-\delta)}-1}{2 \beta(n-1)}-\frac{(n-\delta) \frac{n-1}{n}+\rho}{\rho \beta+(n-1)} \gtreqless 0 \quad \text { if and only if } \frac{n}{n-\delta} \lesseqgtr \beta,
$$

which implies that the intersection point between the curve $C_{2}$ and the satiation locus $1 / \theta$ is located above the intersection point between the curve $C_{3}$ and the satiation locus $1 / \theta$ in all figures except for Fig.9.

${ }^{6}$ The curve $C_{3}$ in (12) is negatively sloped if

$$
\beta<\frac{2 n-1}{n-\delta-\rho}
$$

However, it would be uncertain whether this condition is satisfied in Figs. 2, 3, and 4, although there is certainly some range of $\beta$ in Figs. 3 and 5 which satisfied this condition. Even if the curve $C_{3}$ has a positive slope in Fig.2, the essence of the dynamics does not change as long as the intersection point between the curve $C_{2}$ and the satiation locus $1 / \theta$ is located above that between the curve $C_{3}$ and the satiation locus $1 / \theta$. Footnote 5 shows that this condition holds in all figures except for Fig.9. 


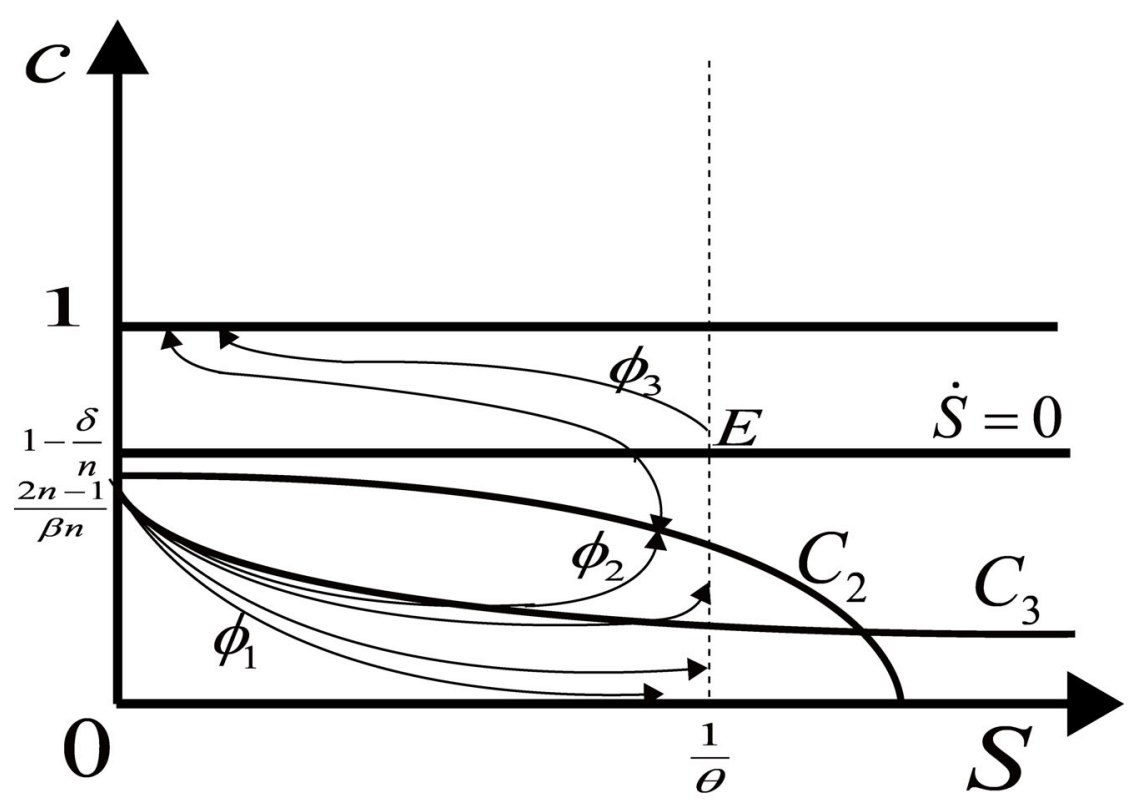

Figure $2: \beta \geq \frac{2 n}{n-\delta}$

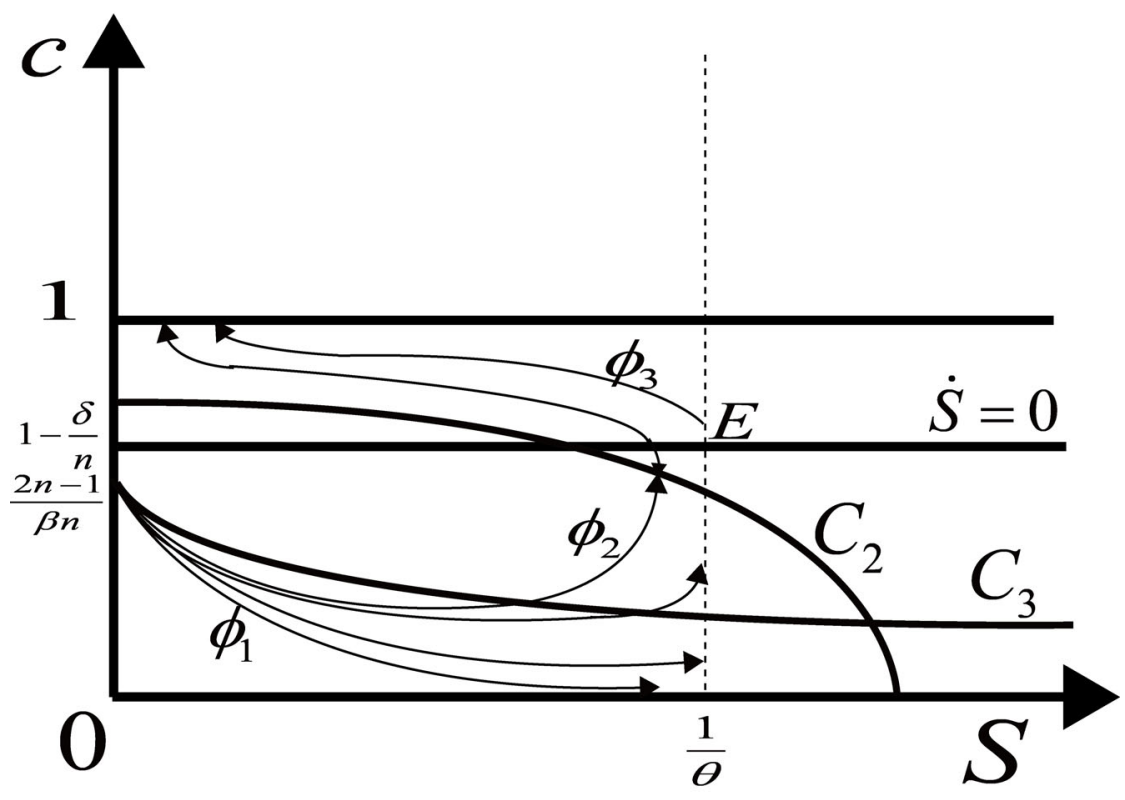

Figure $3: \frac{2 n}{n-\delta} \geq \beta \geq \max \left\{\frac{2 n-1}{n-\delta}, \frac{(n-\delta)(2 n-1)-n}{n(n-1)}\right\}$ 


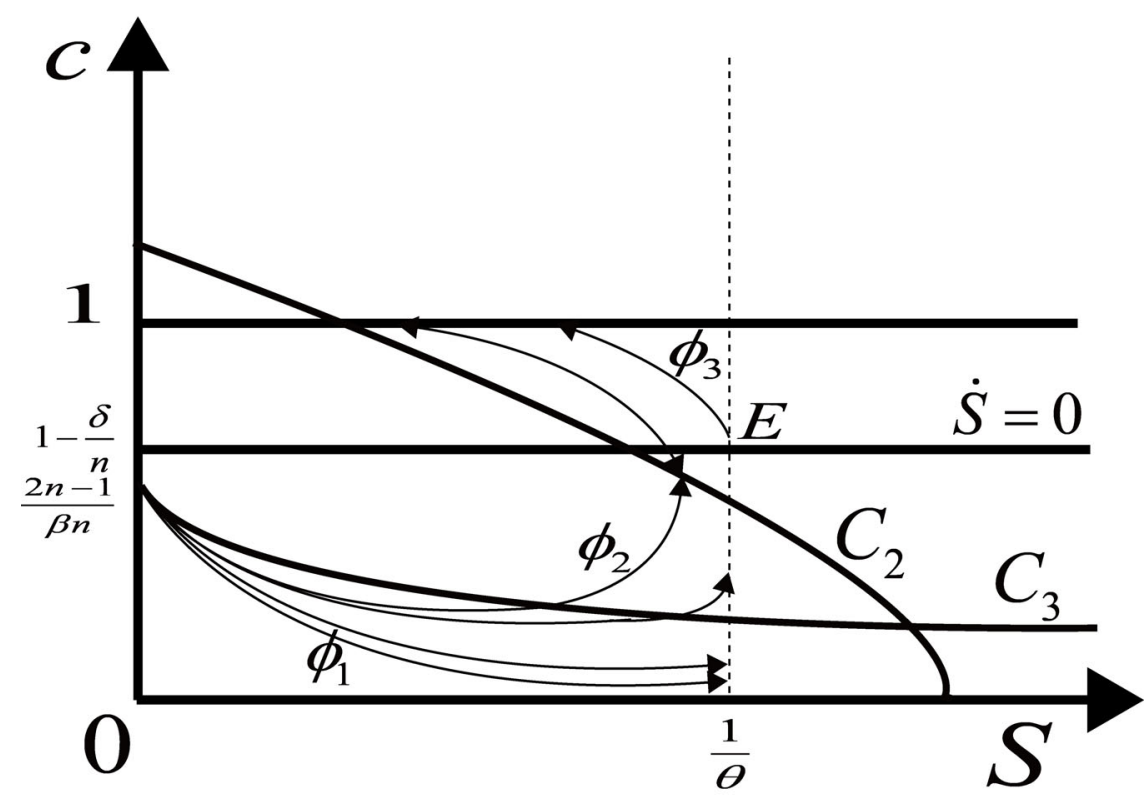

Figure 4: $\frac{(n-\delta)(2 n-1)-n}{n(n-1)} \geq \beta \geq \frac{2 n-1}{n-\delta}$

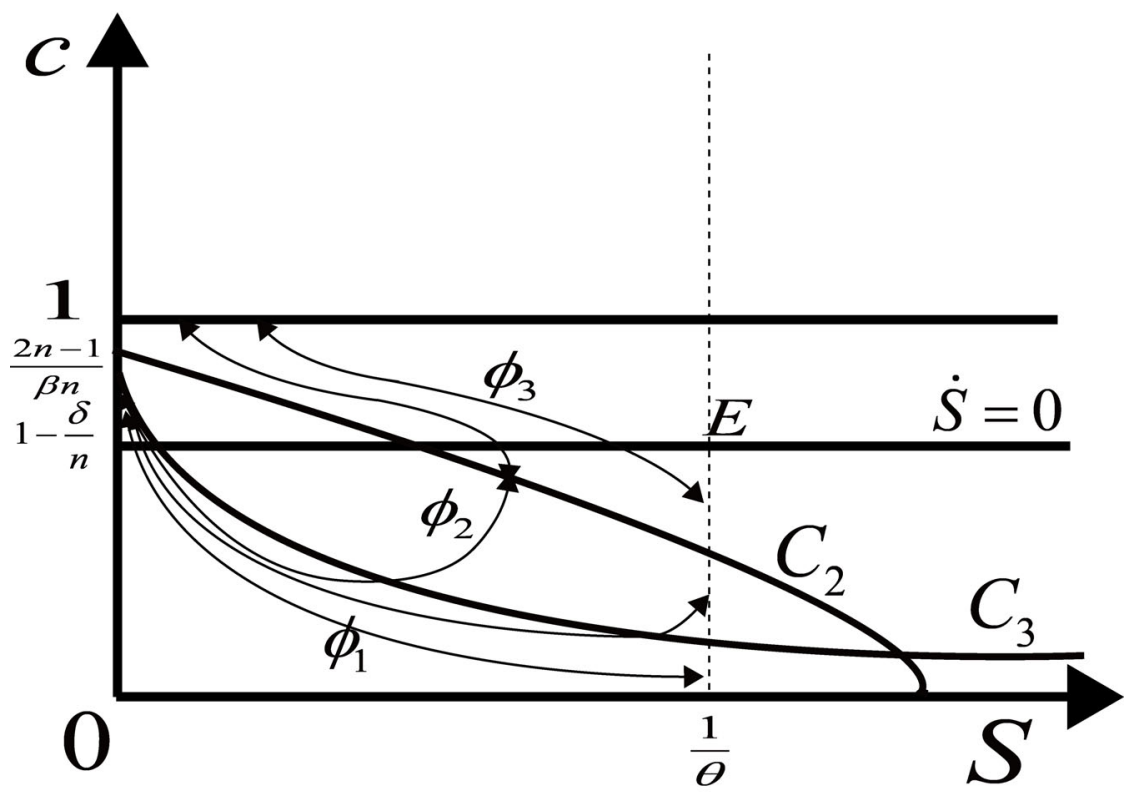

Figure 5: $\frac{2 n-1}{n-\delta} \geq \beta \geq \frac{2(n-1)+(1 / n)}{n-\delta}$ 


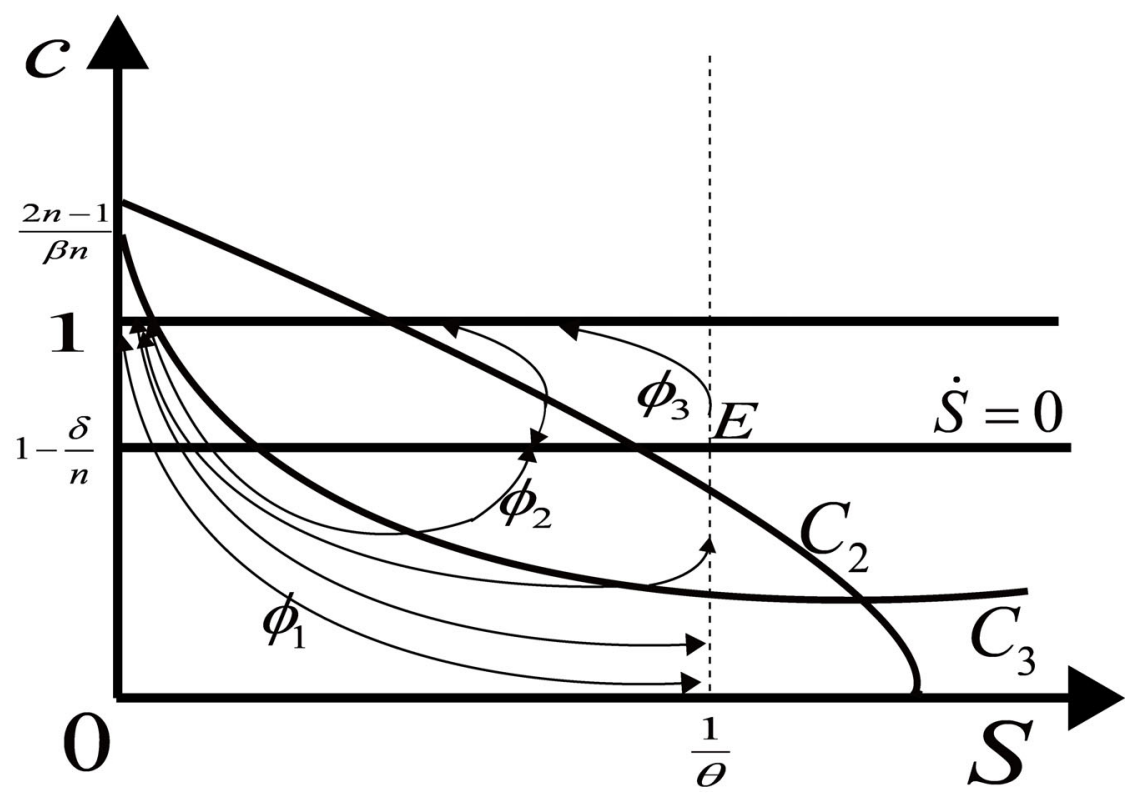

Figure 6: $\min \left\{\frac{2 n-1}{n}, \frac{(n-\delta)(2 n-1)-n}{n(n-1)}\right\} \geq \beta \geq \frac{n}{n-\delta}$

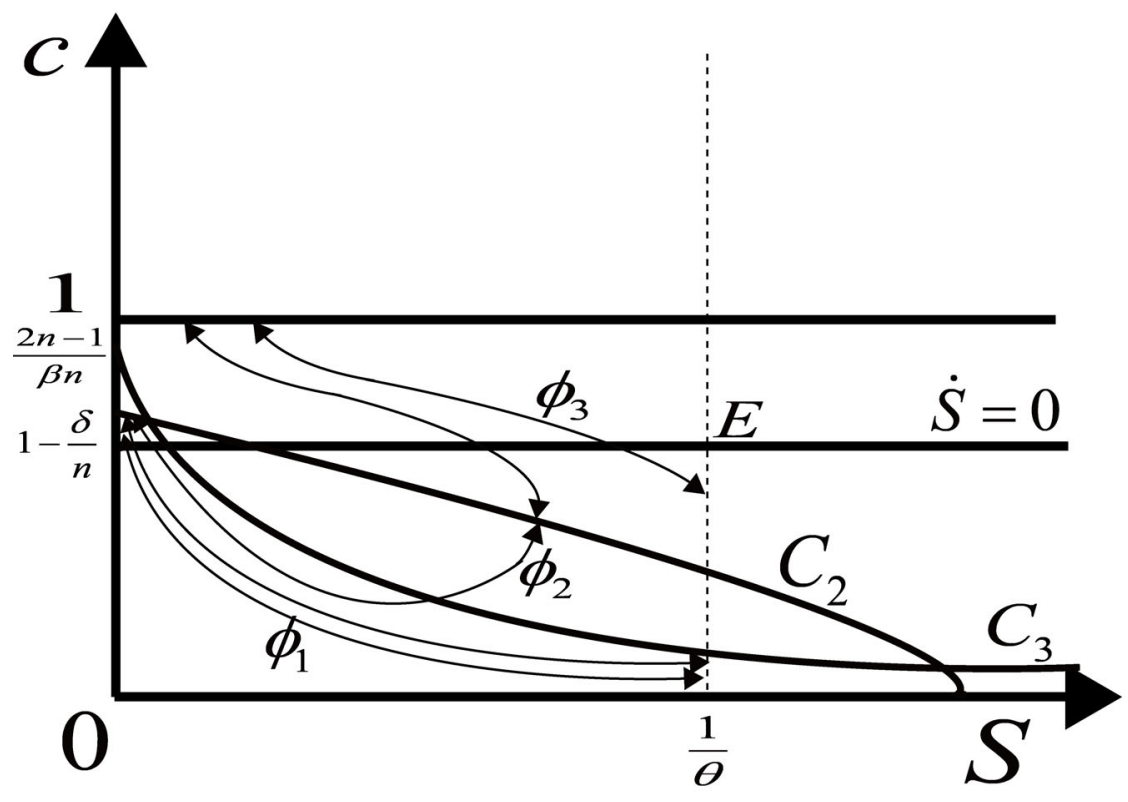

Figure 7: $\frac{2(n-1)+(1 / n)}{n-\delta} \geq \beta \geq \max \left\{\frac{(n-\delta)(2 n-1)-n}{n(n-1)}, \frac{2 n-1}{n}, \frac{n}{n-\delta}\right\}$ 


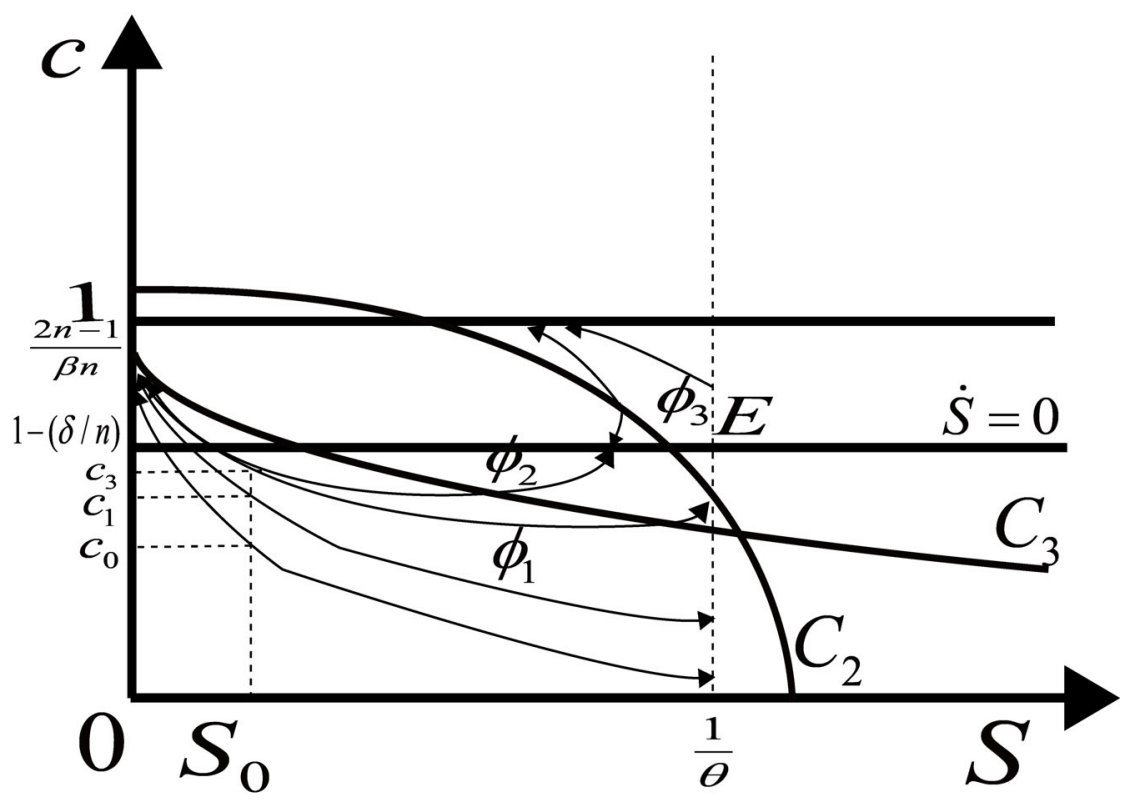

Figure 8: $\frac{(n-\delta)(2 n-1)-n}{n(n-1)} \geq \beta \geq \max \left\{\frac{2 n-1}{n}, \frac{n}{n-\delta}\right\}$

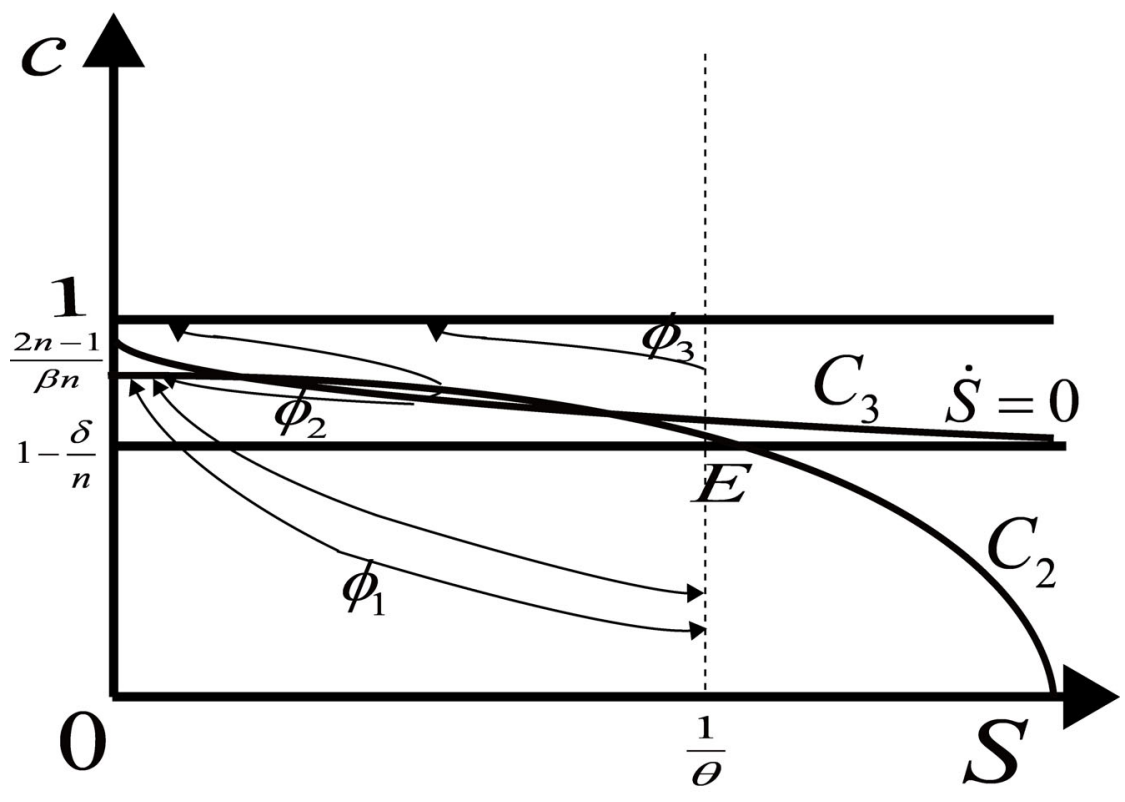

Figure 9: $\frac{n}{n-\delta} \geq \beta \geq \max \left\{\frac{2 n-1}{n}, \frac{(n-\delta)(2 n-1)-n}{n(n-1)}\right\}$ 


\subsection{Refining Candidate Strategies}

In this subsection, we will test whether these candidate strategies satisfy the sufficiency conditions in Theorem 4.4 of Dockner et al. (2000). More specifically, the globally defined MPE must satisfy the following three requirements: First, the strategies must be defined over the entire feasible state space (such as $S \in[0,1 / \theta]$ in the present model) and not only for a (i.e., any) subset. Rowat (2006) claims that we have to take the definition of subgame perfection seriously, since we cannot explain how an initial condition of the state variable was reached in the first place. To show how this requirement is used to refine candidate strategies, we consider representatives of those integral curves displayed by Figs. $2-9$ that are categorized into three typical types of the families of strategies designated by the integral curves $\phi_{j}, j=1,2$, and $3 .^{7}$ The arrows attached to those integral curves indicate the evolution of $S$ and $c$ over time. Applying the first requirement, the strategies $\phi_{2}$ can be ruled out, because they do not cover the whole range of $S$ (i.e., $S \in[0,1 / \theta]$ ) as seen in Figs. $2-9$. It appears that the strategies $\phi_{2}$ could be extended to the entire domain of $S$ by patching them with the corner strategy $\phi=1$ whenever they hit the budget constraint $c=1$, as Rowat (2004) did; however, the extended strategy of $\phi_{2}$ cannot cover the whole rage of $S \in[0,1 / \theta]$ in Figs. $2-9$, because $\phi=1$ always leads the stock of social capital to monotonically decline to zero. On the other hand, although it also seems that the strategies $\phi_{3}$ could cover the whole range of $S \in[0,1 / \theta]$ by such an extension, that may not be the case. Whenever $\phi_{3}$ hits the budget constraint $c=1, \phi=1$ should be binding along the constraint $c=1$. Hence, we need to check whether the condition for the corner strategy $\phi=1$ in (7) is binding or not along this corner component. ${ }^{8}$ The following lemma provides a sufficient condition:

Lemma 1 The corner strategy $\phi=1$ is binding if the value function (B.2) in Appendix $B$ possesses a zero constant of integration and the domain of the state variable $S$ is given by $\left[0, \frac{1}{\theta}\left(1-\frac{n(\beta-1)}{n-1}\right)\right]$.

\footnotetext{
${ }^{7}$ Note that the distinction between the strategies $\phi_{1}$, and $\phi_{2}$, lies in the fact that the strategies $\phi_{1}$ reach the satiation locus $1 / \theta$, but the strategies $\phi_{2}$ do not.

${ }^{8}$ Note that the strategies $\phi_{1}$ never hit the horizontal axis, because the marginal utility of consumption goes to infinity when consumption approaches zero due to the log utility function, which ensures that consumption levels always remain positive. Hence, we need not to take into account the non-negative constraint $c \geq 0$.
} 
The proof of Lemma 1 is relegated to Appendix B. Lemma 1 implies that only when $\beta<(2 n-1) / n$, the extreme right end of the above interval, $\frac{1}{\theta}\left(1-\frac{n(\beta-1)}{n-1}\right)$, is positive. Inspection of Figs $2-9$ immediately reveals that all figures except for Figs. 5 and 6 fail to satisfy this condition. In Figs. 5 and 6 , on the other hand, all interior strategies $\phi_{3}$ starting from any point lying on the satiation locus $1 / \theta$ may either cross the locus $C_{2}$ or hit the budget constraint $c=1$ before reaching the locus $C_{2}$. Any interior strategy $\phi_{3}$ crossing the locus $C_{2}$ does not qualify as a MPE strategy, because it does not satisfy the second requirement which will be stated below. If the strategies $\phi_{3}$ hit the constraint $c=1$, they should be connected with the binding corner strategy $\phi=1$. We can demonstrate the following lemma:

\section{Lemma 2}

(i) he strategies $\phi_{3}$ in Figs. 4 and 5 may be connected with the binding corner strategy $\phi=1$, whereas they appearing in other figures cannot be connected with the the binding corner strategy $\phi=1$.

(ii) The strategies $\phi_{1}$ in Fig. 6 can be connected with the binding corner strategy $\phi=1$.

The proof of Lemma 2 is relegated to Appendix C. The strategies $\phi_{3}$ can be connected with the binding corner strategy $\phi=1$, when the right end point $\frac{1}{\theta}\left(1-\frac{n(\beta-1)}{n-1}\right)$ is located to the right of the non-invertibility locus $C_{2}$. In addition, this right end point should be positive. These two conditions may be satisfied in Figs. 5 and 6 , whereas they are not in other figures. In contrast, since the strategies $\phi_{1}$ in Fig. 6 hit the constraint $c=1$ to the left of the curve $C_{3}$, they can be always connected with the binding corner strategy $\phi=1$. This is partly because all of the strategies $\phi_{1}$ in Fig. 6 hit the constraint $c=1$ to the left of the curve $C_{3}$ which is situated on the left side of the locus $C_{3}$, partly because the above right end point is located to the right of the locus $C_{2}$.

The second requirement is that the MPE strategies should be continuous over the entire domain of a state variable, i.e., $S \in[0,1 / \theta] .{ }^{9}$ More specifically, whenever the strategies cross the non-invertibility locus $C_{2}$, they cease to be continuous at that crossing point, and thus such strategies should be eliminated as globally defined MPE ones. For this reason, the

\footnotetext{
${ }^{9}$ Dockner and Soger (1996) have derived MPE strategies which are discontinuous but which generate a continuous value function. We will impose the assumption of continuity as a requirement for MPE strategies to further refine MPE strategies following Rowat (2007) and Wirl (2007).
} 
strategies $\phi_{2}$ displayed in Figs. $2-9$ are disqualified if they pass the locus $C_{2}$. For the same reason, the strategies $\phi_{1}$ displayed in Figs.7 and 9 are disqualified because they cross the non-invertibility locus $C_{2}$ before reaching the singular point $(0,(2 n-1) / \beta n)$. Note also that if the strategies $\phi_{3}$ were to cross the locus $C_{2}$, they are also disqualified.

The third requirement is that the candidate strategy should be stable in the sense that it converges to the steady state from any initial value of a state variable (see, e.g., Tsutsui and Mino, 1990, Wirl, 2007). However, as shown in Figs. 2-9, there is no stable interior steady state point on the steady state line $\dot{S}=0$. The stability property is needed to guarantee the bounded value function in (5). In our setting, the value functions associated with the solution paths which go to either $S=0$ or $S=1 / \theta$ are all bounded in conjunction with appropriate choices of an integral constant, mainly because the domains for a state variable and a control variable are both bounded.

In spite of these stringent requirements, there are still multiple (or a continuum of) MPE $\phi_{1}$ strategies that pass all three tests. The $\phi_{1}$ strategies can be divided into two types. The first type of them monotonically converges to a point lying on the satiation locus $1 / \theta$ as seen in Figs. 2, 3, and 4; the second type goes to either zero social capital stock (i.e., the singular point $(0,(2 n-1) / \beta n))$ or the satiation level of social capital stock (i.e., $S=1 / \theta)$, depending on the initial level of social capital stock as well as the initial choice of consumption associated with that initial stock as seen in Figs. 5, 6, and 8.

Taken together, the results are summarized as follows:

Proposition 1 Consider the differential game $\Gamma\left(Z_{0}, 0\right)$ :

(i) There exist a continuum of MPE strategies which monotonically go to a satiation level of social capital from any initial stock.

(ii) If there exists an interior steady state, it is globally unstable, and, moreover, there exist a continuum of MPE strategies starting from any initial social capital stock less (greater) than the level associated with the unstable steady state which go to zero social capital (correspondingly, the satiation level of social capital).

(iii) There may exist a continuum of MPE strategies which monotonically go to zero social capital from any initial stock. 
Regarding Proposition 1, several remarks are in order. First, Proposition 1 implies that there may be multiple or a continuum of MPE strategies $\phi_{1}$ (possibly, $\phi_{3}$ ) displayed in Figs. 2, $3,4,5,6$, and 8 . The existence of multiple MPE strategies would be consistent with the results of Itaya and Shimomura (2001), and Rubio and Casino (2002) which also show a multiplicity of MPE strategies as well as the corresponding uncountable many long-run equilibria. This similarity between our model and theirs stems from the boundedness of the entire space of a state variable imposed by exogenous restrictions such as institutional constraints including law, social norms, governmental regulations, limited resources, the satiation level of a state variable in the present model, and so on. This is because a bounded state space is more likely to allow for multiple paths which entail bounded value functions and thus satisfy the transversality condition.

Second, there exist a continuum of solution paths starting from any initial stock of social capital which all lead to a satiation level of social capital stock for relatively higher values of $\beta$ such as in Figs. 2, 3, and 4. It is straightforward to show that a decrease in $\beta$ results in an upward shift of the curve $C_{3}$. As a result, the curve $C_{3}$ may cross the steady state line within the interior of $[0,1 / \theta]$, as illustrated in Figs. 5, 6, and 8, so that there emerges an unstable steady state which entails two long run equilibria. Moreover, the lower the values of $\beta$, the further to the right the intersection between the locus $C_{3}$ and the steady state line moves, and thus the larger the range where the initial level of social capital is less than its steady state level (i.e., the basin of attraction of zero social capital). This implies that for lower values of $\beta$ it is more likely that social capital declines to zero. The intuition is simple. Since the parameter $\beta$ represents the disutility cost of labor supply, lower values of $\beta$ induce the individuals to substitute labor supply for social activities (i.e., reducing $a$ ); consequently, the increased labor supply raises consumption due to the budget constraint (3) (i.e., a positive income effect), thus causing overconsumption.

Third, for relatively lower values of $\beta$ there may be two long-run equilibria which are led by the MPE strategy $\phi_{1}$ and $\phi_{2}$ in Figs. 5, 6, and 8. In this case, the society may potentially go to either of the long run equilibria characterized by a null social capital stock and by a satiation level of social capital, depending on the initial levels of social capital as 
well as the choice of initial consumption levels associated with a given initial stock of social capital. The dependence on the choices of multiple initial consumption levels stems from the fact that even if the path of social capital accumulation starts from the same initial social capital stock, individuals are confronted by the choice of initial consumption levels associated with multiple (a continuum of) MPE strategies $\phi_{1}$ at the outset of the game. As a result, social capital accumulation can follow different paths associated with different choices of initial consumption (for example, $c_{0}, c_{1}$, and $c_{2}$ corresponding to different MPE strategies $\phi_{1}$, respectively, at the initial social capital stock $S_{0}$, as illustrated in Fig. 6), thus leading to different long-run equilibria characterized by different consumption levels. In short, in Figs, $2,3,4,5,6$, and 8 the dynamics of social capital accumulation characterized by the strategies $\phi_{1}$ (possibly, including some of the MPE strategies $\phi_{3}$ if they exist) is path dependent.

Fourth, as stated above, the social-capital affluent society by itself is able to autonomously attain the satiation level of social capital, whereas the social capital-poor society may be caught in a social-capital poverty trap due to the presence of strong status-seeking preferences enhanced by the free-riding incentive to investment in social capital. In addition, whether the poverty tap may or may not emerge in the long run also hinges on the magnitude of the parameters $\beta, n$, and $\delta$. Graphically, since a lower disutility of labour $(\beta)$ causes an upward shift of the curve $C_{3}$ thereby moving the intersection between the curve $C_{3}$ and the steady state line towards the right. This ends up expanding the basin of attraction of the poverty tap. Larger numbers of individuals $(n)$ tend to enhance the intensity of statusseeking competition, thus stimulating consumption. This is illustrated as an upward shift of the curve $C_{3}$. However, increasing community's members brings more endowments to that community and thus results in more contributions to social capital (which we call the endowment effect), which is illustrated in an upward shift of the steady state line also. Hence, it would be uncertain which direction the intersection between these two loci moves. ${ }^{10} \mathrm{~A}$ larger depreciation rate $(\delta)$ unambiguously shifts that intersection point to the right, thereby

\footnotetext{
${ }^{10}$ The level of social capital stock at the interesection between the locus $C_{3}$ and the steady state line is given by

$$
\tilde{S}=\frac{(2-\beta) n+\beta \delta-1}{\theta(n-1)} .
$$
}

Differentiating the above expression yields

$$
\frac{d \tilde{S}}{d n}=\frac{-1+\beta(1-\delta)}{\theta(n-1)} \gtreqless 0 \text { if and only if } \beta \gtreqless \frac{1}{1-\delta} .
$$


expanding the basin of attraction of zero social capital stock.

Fifth, the optimal consumption paths supported by the MPE strategies $\phi_{1}$ in general display a downward sloping curve in the level of social capital stock. This feature obviously reflects the fact that larger (smaller) social capital stocks tend to deter (accelerate) excessive consumption which is caused by status-seeking behavior. In other words, since social capital generates relatively strong complementarities in individual investment for social capital, people who belong to the same groups or communities with more (less) social capital tend to make more (less) investment in social capital. However, as there are negative externalities stemming from status-seeking behavior of other individuals, whether individual's consumption declines or rises as social capital is accumulating or whether underconsumption or overconsumption prevails in the long run depends critically on which effect dominates. If the stock externality from social capital outweighs the consumption externality in conjunction with the free-riding incentive for individual's investment in social capital, the MPE strategies $\phi_{1}$ allow for social capital to accumulate up to its satiation level, as illustrated in Figs. 2, 3, and 4 (including the strategies $\phi_{1}$ which converge to $S=1 / \theta$ in Figs. 5, 6, and 8 ). Conversely, if the former effect is dominated by the latter two effects, consumption is rising as social capital is accumulating and thus overconsumption prevails, which would explain the behavior of the strategies $\phi_{1}$ which converge to zero social capital in Figs. 5, 6, and 8. In this case, the consumption levels associated with those strategies keep rising up to $S=0$. Some of the strategies $\phi_{1}$ eventually display an upward slope when $S$ is close to its satiation level $1 / \theta$. This is because the tendency of overconsumption arises due to decreasing the weight $1-\theta S$, which in turn weakens suppressing overconsumption to the extent that it is not enough to discourage social capital accumulation.

If the society were to be trapped in a poverty trap (i.e., a situation of zero social capital stock), government intervention should be called upon to help the societies escape from the poverty trap. Since the poor social-capital society by itself can never spontaneously and autonomously accumulate social capital, governments and/or outside authorities have to enforce or regulate the members of the society so as to suppress their consumption if consumption is accessibly stimulated by the status-seeking motive. For example, the introduction of conIn particular, if $\beta>1 /(1-\delta)$, the effect on status-seeking competion dominates the endowment effect, thereby shifting the intersection point to the right. 
sumption taxation policy would be a very effective means to depress individual consumption. An increase in the consumption tax rate would be thought of as a higher value of $\beta$ because the tax increase raises the effective price (opportunity cost) of private consumption. ${ }^{11}$ As stated before, when an initial stock of social capital is less than that of the unstable steady state, there is a threshold level of initial consumption, below which the society is able to avoid a path leading to the social poverty trap. Under this circumstance, the government may be willing to coordinate on the initial choices of individual consumption to reduce their consumption by campaigns, education, public announcements, communication, or governmental regulations.

Another policy instrument to promote social capital accumulation is to decrease the rate of depreciation $(\delta)$ through the degree of residential mobility (more broadly, the parameter $\delta$ may be interpreted as the ability of economic agents to preserve existing social connections). To this end, governments may make a community more attractive thereby discouraging residents from exiting it. To do this, governments may build attractive public meeting places such as a community center or pubs, or promote the formation of clubs or social networks (see Glaeser, et al., 2002). Since the decrease in $\delta$ shrinks the basin of attraction of the poverty trap, the social capital poverty trap is less likely to emerge in the long run.

\section{Welfare comparison}

In this section, we first compare the levels of social welfare (or consumption levels) in a hypothetical social planner's economy and in a decentralized economy in which social capital is accumulated through voluntary effort (social activity) made by individuals through time. We also compare the welfare levels attained in the decentralized economy under a satiationlevel and a zero-level (corresponding to a poverty trap) of steady-state social capital.

\footnotetext{
${ }^{11}$ When consumption taxes are introduced, the budget constraint is modified as follows:

$$
(1+\tau) c=l+T r
$$

where $\tau$ represents a consumption tax rate and $\operatorname{Tr}$ represents the transfer income to individuals. Substituting into $l$ in the utility function and setting $\operatorname{Tr}=\tau c$ in order to exclude an income effect, the parameter $\beta$ may be changed into $\beta /(1+\tau)$; the only substitution effect caused by the increased consumption tax rate is effective, thereby depressing the individual's consumption.
} 
The social planner is able to directly control resource allocations in order to fully internalize the negative externality arising from the aspiration for relative consumption. In the planner economy, therefore, the status externality is completely eliminated and the first-best allocation restored. This amounts to maximizing the following utility function over an infinite horizon:

$$
u_{i}=\int_{0}^{\infty}\left[\log c_{i}-\beta l_{i}\right] e^{-\rho t} d t
$$

subject to $c_{i}=l_{i}$, whose optimal solution is given by $c^{P}=1 / \beta<1$. Note not only that the Pareto efficient consumption level remains constant through time, but also that it is less than the steady state consumption level so long as $\beta>n /(n-\delta)^{12}$.

We will compare between the Pareto-efficient level of consumption $(=1 / \beta)$ and the longrun consumption level realized in the decentralized economy with zero social capital or the satiation level of social capital. First, since multiple long run consumption levels can be attained by a continuum of the MPE strategies $\phi_{1}$ in the decentralized economy, as a reference we first consider the long run consumption level corresponding to the intersection point between the curve $C_{3}$ and the satiation locus $1 / \theta$, which is given by

$$
\frac{(n-\delta) \frac{n-1}{n}+\rho}{\rho \beta+(n-1)} \text {. }
$$

It is straightforward to show that

$$
\frac{(n-\delta) \frac{n-1}{n}+\rho}{\rho \beta+(n-1)}-\frac{1}{\beta}=\frac{1}{n \beta}(n-1) \frac{-n+\beta(n-\delta)}{n+\beta \rho-1}>0 \text { if } \beta>\frac{n}{n-\delta} .
$$

Since the above condition is satisfied in all figures, (24) implies that the long run consumption level at $S=1 / \theta$ is higher than the Pareto-efficient one $(=1 / \beta)$. In other words, the decentralized economy can achieve lower consumption levels or the Pareto efficient one by appropriately choosing the MPE strategies $\phi_{1}$ in the decentralized economy.

In Figs. 5 and 8, on the other hand, the consumption level corresponding to the social poverty trap is uniquely determined because the MPE strategies $\phi_{1}$ converge to the singular point $(0,(2 n-1) / \beta n)$. As a result, comparison yields

$$
\frac{2 n-1}{\beta n}-\frac{1}{\beta}=\frac{1}{n \beta}(n-1)>0
$$

\footnotetext{
${ }^{12}$ Although this condition is not satsfied in Fig.9, we ignore this case in what follows because in Fig.9 there are no MPE strategies.
} 
which implies that the consumption level at the social poverty trap (i.e., $S=0$ ) is unambiguously higher than the Pareto-efficient one. In Fig.6, following the MPE strategies $\phi_{1}$ which goes to $S=0$ (possibly, the MPE strategies $\phi_{3}$ in Figs. 5 and 6 , if they exist), the resulting consumption level at $S=0$ is equal to 1 . As a result, it is obviously higher than the Pareto-efficient one $(=1 / \beta<1)$.

Lastly, we compare between the welfare level (i.e., the individual's utility level) at the social poverty trap (i.e., $S=0$ ) and that realized by any of the MPE strategies $\phi_{1}$ at the satiation level of social capital (i.e., $S=1 / \theta$ ). As a reference point, we once again compare between the consumption level at $S=0$ and that evaluated at the intersection point between the curve $C_{3}$ and the satiation locus $1 / \theta$ to yield:

$$
\begin{aligned}
\frac{2 n-1}{\beta n}-\frac{(n-\delta) \frac{n-1}{n}+\rho}{\rho \beta+(n-1)} & =(n-1) \frac{2 n-1+\beta(-n+\delta+\rho)}{n \beta(n+\beta \rho-1)} \gtreqless 0, \\
\text { if and only if } \beta & \lesseqgtr \frac{2 n-1}{n-\delta-\rho} .
\end{aligned}
$$

Since the instantaneous utility function, $\log c-\beta c$, is decreasing in $c$ if $c>1 / \beta$ and taking into account the fact that

$$
\frac{2 n-1}{n-\delta-\rho}-\frac{n}{n-\delta}=\frac{-n+\delta-n \delta+n \rho+n^{2}}{(n-\delta)(n-\delta-\rho)}>0,
$$

we arrive at the conclusion that if $\beta>\frac{2 n-1}{n-\delta-\rho}$, the welfare level evaluated at $S=1 / \theta$ is higher than that the social poverty trap, while if $\frac{2 n-1}{n-\delta-\rho}>\beta>\frac{n}{n-\delta}$, the opposite holds. This result implies that since the consumption level at $S=1 / \theta$ is less than the consumption level at $S=0$ under the condition in (25), the welfare level at $S=1 / \theta$ will be higher than that at $S=0$. Moreover, in the decentralized economy it is possible that individuals choose further lower consumption levels or the consumption level which is closer to the Pareto efficient one. In either case, the decentralized economy is able to attain higher welfare levels at $S=1 / \theta$ compared to that at the social poverty trap (i.e., $S=0$ ), irrespective of what condition in (25) holds.

To sum up, we have the following lemma:

Proposition 2 Assume $\beta>n /(n-\delta)$. 
(i) The long run consumption levels corresponding to the satiation level of social capital in the decentralized economy may or may not be higher than the Pareto-efficient one, while the long-run consumption level realized in the social poverty trap is higher than the Pareto-efficient one.

(ii) There certainly exist the MPE strategies that realize higher welfare levels at the satiation level of social capital compared to that at the social poverty trap in the decentralized economy.

\section{Concluding Remarks}

This paper proposes a novel formal framework to model social capital as an aggregate state variable that alleviates unsociable/sub-optimal behavior in a dynamic economy. By clarifying a concept that has been the subject of ambiguity, our analysis yields novel insights and advances on existing literature. To capture inefficiencies and highlight the benefits (positive externalities) of social capital, we adopt a utility function that exhibits status-seeking preferences (alternatively known as "keeping up with the Joneses"), which may potentially induce prisoner's dilemma type of behavior. But status preferences are only one example of negative externalities; our framework is compatible with a broader set of unsociable (i.e., inefficient) behavior instead of status. We use differential game theory to model the intertemporal formation of social capital across individuals as a MPE in which every individual takes into account the negative externality arising from status-seeking behavior as well as the free-riding behavior associated with social capital accumulation. In spite of the presence of these negative externalities, this study reveals a possibility that the formation of social capital spontaneously and autonomously take place among a finite number of rational and forward-looking individuals, which ends up deterring overconsumption and thus leads to a social-capital affluent society being Pareto-superior to a social-capital poor one.

The first message of this paper is that it is more likely than expected that individuals are willing to accumulate social capital up to its satiation level without any intervention of outside enforcers such as governments. That is, there would be a continuum of MPE strategies which may lead the economy to spontaneously attain a continuum of long-run equilibria characterized by the satiation level of social capital combined with a wide range of long-run 
consumption levels. Nevertheless, if the disutility of labor supply or the wage rate takes low values or the depreciation rate of social capital takes higher values, there may be two pathdependent long-run equilibria, one of which realizes the satiation level of social capital and the other of which leads to a social poverty trap. In other words, there is a possibility that fully rational and forward-looking individuals may lead to the undesirable social capital poverty trap characterized by zero social capital, when the negative externality arising from statusseeking preferences is strong enough to outweigh the positive externality arising from social capital accumulation. The former effect is enhanced by incomplete collective internalization of the positive externality inherent in the formation of social capital due to its public good characteristics (i.e., free-riding incentives for individual investment in social capital). In short, whether the game for social capital accumulation is a prisoner's dilemma game or a coordination game would depend on which effect dominates. The initial choice of individual consumption in conjunction with the historically given initial stock of social capital also matter in determining which effect dominates and thus which long-run outcome emerges, when multiple MPE strategies are present. In the decentralized economy, individuals are usually unable to coordinate on a simple range of actions that would make all members of a community better off or entail a Pareto-dominated equilibria; hence, the so-called coordination failure occurs. These results would be consistent with the casual observations that the level of social activity and the stock level of social capital are sometimes different to a large extent between similar communities in terms of economic fundamentals. Another message is that our result of multiple equilibria stands in a sharp contrast with results of earlier literature such as Growiec and Growiec (2014), and Varvarigos and Xin (2015); in their models, a multiplicity of long-run equilibria arise from the nonconvexity of social capital accumulation, whereas in our model the multiplicity arises from the strategic interaction through the above-mentioned externalities without nonconvexity of a particular function such as a social capital formation function or trust formation function. To the best of our knowledge, this key finding of this study is a new insight in the literature.

A third message is that the present analysis would provide certain policy recommendations, when society is captured in a social poverty trap or in order to escape from the above-mentioned coordination failure. In order to eliminate inefficiently high consumption and thus to promote social capital accumulation, the government needs to control individ- 
ual consumption by restricting the number of community members, imposing consumption taxes, coordinating their choices of initial consumption levels, or enhancing the attractiveness of social capital to prevent from exiting of residents. The last policy can be implemented through providing a private incentive to accumulate social capital or the motive of warm-glow giving advocated by Andreoni (1990). As a consequence, the payoff structure of the present prisoner's dilemma-type game may be transformed into that of a coordination game. Alternatively, to resolve the problem of coordination failure, voluntary pre-play communication could be substitutes for government intervention. Nevertheless, to increase an opportunity of communication among individuals, governments also play a certain role in providing public meeting places and financial aid for NPO's (or individuals) which engage in volunteering and promote civic engagement in local regions, and laying the physical infrastructure for education.

The model presented in this paper can be developed further in several directions. First, the weight function put on status-seeking preferences could be relaxed so as to allow for a whole domain of $R_{+}$; for example, negative exponential functions. Even under this extended setting, it would be questionable whether globally defined MPE strategies over $R_{+}$may survive, because it is highly likely that they will ultimately cross the non-invertiblity curve within the feasible domain of a state variable. Nevertheless, with the help of Rowat's patching method, it might be possible to apply the concept of globally defined MPE strategies as in the present analysis. Second, introducing asymmetric agents into the present model would yield more complicated dynamics, although this extension is the most interesting generalization of the present model. Such an asymmetric model could allow for the emergence of the component representing status-seeking preferences in equilibrium because the ratio between individual consumption and average consumption is not equal to 1 and thus no longer vanishes even under logarithmic preferences. This might potentially allow for a variety of MPE strategies and richer outcomes. Third, social capital accumulation itself allows individuals to resolve or mitigate the problem of coordination failure, because social capital could be viewed as a social structure that facilitates coordination and cooperation. We should, therefore, expect that members of communities with high social capital would be more able and/or more willing to reach mutual understanding and agreements, or to share common opinions, values, beliefs, and social norms. For this reason, we need to provide a formal model to clarify how social 
capital affects the ability or incentive of aligning expectations of private agents when choosing their initial consumption levels. Fourth, Putnam (2000) has made a distinction between bridging social capital in which bonds of connectedness are formed across diverse social groups, and bonding social capital that cements only homogenous groups. The endogenous choices of individuals would be informative on how much time they spend on enhancing closed interpersonal networks, which contributes to accumulating bonding social capital, and/or to the formation of open interpersonal networks which contributes to accumulating bridging social capital.

\section{Appendix A: Deviation for (11)}

We once again differentiae $(7)$ (i.e., $V_{i}^{\prime}(S)=\frac{1}{c_{i}(S) S}\left[1-\beta c_{i}(S)+\left(1-\theta_{i} S\right) \frac{\sum_{j=1, j \neq i}^{n} c_{j}(S)}{C(S)}\right]$ ) with respect to $S$ and using symmetry (i.e., $\sum_{j=1, j \neq i}^{n} c_{j}^{\prime}(S) C(S)-\sum_{j=1, j \neq i}^{n} c_{j}(S) C^{\prime}(S)=$ $\left.(n-1) c^{\prime}(S) n c(S)-(n-1) c(S) n c^{\prime}(S)=0\right)$, we obtain

$$
V^{\prime \prime}(S)=\frac{1}{c(S) S}\left[-\beta c^{\prime}(S)-\theta \frac{n-1}{n}-\left(1-\beta c(S)+(1-\theta S) \frac{n-1}{n}\right)\left(\frac{c^{\prime}(S)}{c(S)}+S^{-1}\right)\right] .
$$

Substituting (9) and (A.1) into (8) and rearrangement yields

$$
\begin{gathered}
{\left[\rho+\delta-n(1-c(S))+S(n-1) c^{\prime}(S)\right] \frac{1}{c(S) S}\left[1-\beta c(S)+(1-\theta S) \frac{n-1}{n}\right]=} \\
+\frac{c^{\prime}(S)}{c(S)}(1-\theta S) \frac{n-1}{n}= \\
\frac{1}{c(S) S}\left[-\beta c^{\prime}(S)-\theta \frac{n-1}{n}-\left(1-\beta c(S)+(1-\theta S) \frac{n-1}{n}\right)\left(\frac{c^{\prime}(S)}{c(S)}+S^{-1}\right)\right] . \\
{[S n(1-c(S))-\delta S] .}
\end{gathered}
$$

After multiplying both sides by $c(S) S$, we collect the terms related to $c^{\prime}(S)$ on the left-hand side of (A.3) and rearrange terms to yield

$$
\begin{gathered}
c^{\prime}(S)\left[\frac{1}{c(S)}((n-c(S))-\delta)\left(1-\beta c(S)+(1-\theta S) \frac{n-1}{n}\right)\right. \\
\left.+(1-\theta S) \frac{n-1}{n}+\beta(n(1-c(S))-\delta)\right]= \\
-\rho\left[1-\beta c(S)+(1-\theta S) \frac{n-1}{n}\right] S^{-1}-\theta \frac{n-1}{n}[n(1-c(S))-\delta],
\end{gathered}
$$

which gives us (11). 


\section{Appendix B: Proof of Lemma 1}

Proof. When all individuals play $\phi=1$, the HJB equation (6) becomes

$$
\rho V(S)=-\beta+V^{\prime}(S)(-\delta S)
$$

Integrating (B.1) yields

$$
V(S)=-\frac{\beta}{\rho}+A S^{-\frac{\rho}{\delta}},
$$

where $A$ is an arbitrary constant of integration. In follows from (B.2) that when $A>0$, $\lim _{S \rightarrow 0} V(S)=\infty$. Since we have to rule out the case of the unbounded value function, the case of $A>0$ should be eliminated. Similarly, when $A<0$, the value function diverges to minus infinity thus contradicting the bounded value function. Taken together, the only candidate with $A=0$ remains as a possible candidate. In this case, the corner strategy $\phi(S)=1$ must be consistent with (B.3); consequently,

$$
1-\beta+(1-\theta S) \frac{n-1}{n} \geq 0
$$

which can be rewritten as follows:

$$
S \leq \frac{1}{\theta}+\frac{n(1-\beta)}{\theta(n-1)}
$$

which completes the proof.

\section{Appendix C: Proof of Lemma 2}

Proof. To show this, we first identify the value of $S$ corresponding to the intersection between the curve $C_{2}$ and the budget constraint $c=1$, which is given by

$$
\hat{S}=\frac{(n-1) n(2-\beta)+\delta(1-2 n)}{\theta(n-\delta)(n-1)} .
$$

The difference between the above $\hat{S}$ and $\frac{1}{\theta}\left(1-\frac{n(\beta-1)}{n-1}\right)$ is given by

$$
\frac{(n-1) n(2-\beta)+\delta(1-2 n)}{\theta(n-1)(n-\delta)}-\frac{1}{\theta}\left(1-\frac{n(\beta-1)}{n-1}\right)=\frac{n[\beta(1-\delta)-1]}{\theta(n-\delta)(n-1)} \gtreqless 0,
$$

if and only if $\beta \gtreqless 1 /(1-\delta)$. 
In order to connect the strategies $\phi_{3}$ with the binding corner strategy $\phi=1$, the extreme right end point of the range $\frac{1}{\theta}\left(1-\frac{n(\beta-1)}{n-1}\right)$ in Lemma 1 should be located to the right of the non-invertible locus $C_{2}$. It holds when the sign of $(\mathrm{C} 1)$ is negative (i.e., $\beta<1 /(1-\delta)$ ). Combining this condition with the condition in Lemma 1 yields

$$
\beta<\min \left[\frac{2 n-1}{n}, \frac{1}{1-\delta}\right]
$$

Inspection of all figures reveals that condition (C2) may be satisfied only in Figs.5 and 6, although it is definitely not in all figures except for Figs.5 and 6. On the other hand, the curve $C_{3}$ intersects with the constraint $c=1$ at

$$
\check{S}=\frac{\rho}{\theta} \frac{2 n-n \beta-1}{(\delta+\rho)(n-1)},
$$

which implies that $\check{S}$ is positive if $\beta<(2 n-1) / n$. Moreover, we can confirm that the above intersection point is located to the right of the above right end point

$$
\frac{\rho}{\theta} \frac{2 n-n \beta-1}{(\delta+\rho)(n-1)}-\frac{1}{\theta}\left(1-\frac{n(\beta-1)}{n-1}\right)=\delta \frac{-2 n+n \beta+1}{\theta(\delta+\rho)(n-1)}<0
$$

when $\beta<(2 n-1) / n$. Since the strategies $\phi_{1}$ always hit the constraint $c=1$ to the left of the locus $C_{3}$ in Fig.6, condition (C2) is satisfied; consequently, it is always possible to connect them with the binding corner strategy $\phi=1$.

\section{References}

Alonso-Carrera, J., Caballé, J., and X. Raurich (2004), "Consumption externalities, habit formation and equilibrium efficiency," Scandinavian Journal of Economics 106, 231251.

Alvarez-Cuadrado, Monteiro, F., G., and S. Turnovsky (2004), "Habit formation, catching up with the Joneses, and economic growth," Journal of Economic Growth 9, 47-80.

Andreoni, J. (1990), "Impure altruism and donations to public goods: A theory of warmglow giving," Economic Journal 100, 464-477.

Antoci, A., Sacco, P.L., and P. Vanin (2007), "Social capital accumulation and the evolution of social participation," Journal of Socio-Economics 36, 128-143. 
Bowles, S., Gintis, H. (2002), "Social capital and community governance," Economic Journal 112, 419-436.

Chou, Y. K. (2006), "Three simple models of social capital and economic growth," Journal of Socio-Economics 35, 889-912.

Coleman, J. S. (1990), The Foundations of Social Theory, Cambridge, Mass: Harvard University Press.

Djankov, S., Glaeser, E., La Porta, R., Lopez-de-Silanes, F., and A. Shleifer (2003), "The new comparative economics," Journal of Comparative Economics 31, 595-619.

Dockner, E., Jorgensen, S., Long, N.V., Sorger, G. (2000), Differential Games in Economics and Management Science, Cambridge University Press.

Durlauf, S. N. (2002), "On the empirics of social capital," Economic Journal 112, 459-479.

Durlauf, S.N., and M. Fafchamps (2005), "Social capital," in Handbook of Economic Growth, vol 1, North Holland, Amsterdam.

Fukuyama, F. (1997), Social capital, Tanner Lecture on Human Values.

Futagami, K., and A. Shibata (1998), "Keeping one step ahead of the Joneses: Status, the distribution of wealth and long run growth," Journal of Economic Behavior and Organization 36, 109-126.

Glaeser, E. L., D. Laibson, and B. Sacerdote, B "An economic approach to social capital," Economic Journal 112, 437-458.

Grootaert, C., van Bastelear, T. (Eds.) (2002), The Role of Social Capital in Development: An Empirical Assessment, Cambridge University Press, Cambridge.

Growiec, K., and J. Growiec (2014), "Social capital, trust, and multiple equilibria in economic performance," Macroeconomic Dynamics 18, 282-315.

Guiso, L., Sapienza, P., and L. Zingales (2004), "The role of social capital in financial development," American Economic Review 94, 526-556. 
Guiso, L., Sapienza, P., and L. Zingales (2010), "Civic capital as the missing link," J. Benhabib, A. Bisisn, and M. Jackson, eds, 417-480, Handbook of Social Economics, vol.1A, Amsterdam: North Holland.

Helliwell, J. F., Aknin, L.B., Shiplett, H., Huang, H., and S. Wang (2017), "Social capital and prosocial behavior as sources of well-being," NBER Working Paper No. 23761.

Itaya, J., and K. Shimomura (2001), "A dynamic conjectural variations model in the private provision of public goods: A differential game approach," Journal of Public Economics 81, 153-172.

Knack, S., and P. Keefer (1997), "Does social capital have an economic payoff: A cross country investigation," Quarterly Journal of Economics 112, 1251-1288.

Knack, S., and P. Zak (1999), "Trust and growth," Working Paper, Claremont University.

LaPorta, Lopez-de-Silanes, Shleifer, A., and R.Vishny (1999), "The quality of government," Journal of Law, Economics and Organization 15, 222-279.

Putnam, R., R., Leonardi and R. Nanetti (1993), Making Democracy Work, Civic Traditions in Modern Italy, Princeton: Princeton University Press.

Putnam, R., D. (2000), Bowling Alone: The Collapse and Revival of American Community, New York: Simon and Schuster.

Routledge, B.R., and J. von Amsberg (2002), "Social capital and growth," Journal of Monetary Economics 50, 167-193.

Rowat, C. (2007), "Nonlinear strategies in a linear quadratic differential game with bounded controls," Journal of Economic Dynamics and Control 31, 3179-3202.

Rubio, S.J., and B. Casino (2002), "A note on cooperative versus non-cooperative strategies in international pollution control," Resource and Energy Economics 24, 251-261.

Solow, R.M. (2000), Growth Theory: An Exposition, Oxford University Press, Oxford.

Tabellini, G. (2008), "The scope of cooperation: Values and incentives," Quarterly Journal of Economics 123, 905-950. 
Tsoukis, C. (2007), "Keeping up with the Joneses, growth, and distribution," Scottish Journal of Political Economy 54 575-600.

Tsoukis, C., and F. Tournemaine (2013), "Status in a canonical macro model: Labour supply, growth, and inequality," The Manchester School 81, 65-92.

Tsutsui, S., and M. Mino (1990), "Nonlinear strategies in dynamic duopolistic competition with sticky prices," Journal of Economic Theory 52, 136-161.

Varvarigos, D., and G. Xin (2015), "Social interactions, the evolution of trust, and economic growth," Working Paper No. 15/05, University of Leicester.

Wirl, F. (2007), "Do multiple Nash equilibria in Markov strategies mitigate the tragedy of the commons?" Journal of Economic Dynamics and Control 31, 3723-3740. 